\title{
Numerical Study of an Individual Taylor Bubble Drifting through Stagnant Liquid in an Inclined Pipe
}

\author{
$\underline{\text { E.Z. Massoud }}^{1,2}$, Q. Xiao' ${ }^{1}$ H.A. El-Gamal ${ }^{3}$ \\ ${ }^{I}$ Department of Naval Architecture, Ocean and Marine Engineering, University of Strathclyde, Glasgow G4 \\ OLZ, Scotland, UK \\ ${ }^{2}$ Mechanical Engineering Department, Arab Academy for Science and Technology and Maritime Transport, \\ Alexandria, Egypt. \\ ${ }^{3}$ Mechanical Engineering Department, Faculty of Engineering, Alexandria University, Alexandria, Egypt.
}

\begin{abstract}
The objective of this paper is to investigate the motion of a single Taylor bubble through stagnant Newtonian liquid in an inclined pipe by performing a complete dimensionless treatment followed by an order of magnitude analysis of the terms of equations of motion. The main contribution of this analysis is that Froude, Eötvös and Reynolds numbers are the main physical parameters prompting the dimensionless governing equations for inclination angle up to $\theta \geq 70^{\circ}$. The bubble drift velocity diminishes in inclination angles near the horizontal orientation, and thus the reduced Galilei number is suggested to govern the bubble dynamics. To support the developed logical approach of the problem, the present study employs a CFD study so as to investigate the hydrodynamics of single Taylor bubble drifting through stagnant liquid in an inclined pipe through using the volume-of-fluid (VOF) methodology implemented in the computational fluid dynamics software package, ANSYS Fluent (Release 16.0). The simulation results show good correspondence with the developed dimensionless treatment of the problem. Based on the fact that no bubble propagates in a zero axial pressure gradient horizontal pipe, a simplified model is suggested to solve the challenging problem of the threedimensional Taylor bubble in near-horizontal and horizontal pipes, and thus saving computational resources.
\end{abstract}

Keywords: Taylor bubble; order of magnitude analysis; Inclined pipe; VOF method 


\section{Nomenclature}

D

$f_{m}$

$g$

$g_{x}$

$g_{x}{ }^{*}$

$g_{y}$

$g_{y}{ }^{*}$

$g_{z}$

$g_{z}{ }^{*}$

$h_{B}$

$h / D$

K

$K^{*}$

$L$

$L_{T B}$

$p$

$p^{*}$

$\hat{p}$

$\bar{p}$

$Q$

$R_{T B}$

$t$

$t^{*}$

Pipe diameter (m)

Coefficient 1(Zukoski (1966)) in Table 2

Acceleration due to gravity $\left(\mathrm{m} \mathrm{s}^{-2}\right)$

Acceleration due to gravity in $\mathrm{x}$-direction $\left(\mathrm{m} \mathrm{s}^{-2}\right)$

Dimensionless acceleration due to gravity in $\mathrm{x}$-direction (-)

Acceleration due to gravity in y-direction $\left(\mathrm{m} \mathrm{s}^{-2}\right)$

Dimensionless acceleration due to gravity in y-direction (-)

Acceleration due to gravity in z-direction $\left(\mathrm{m} \mathrm{s}^{-2}\right)$

Dimensionless acceleration due to gravity in z-direction (-)

Distance of Taylor bubble initial shape from the pipe bottom (m)

Dimensionless liquid Height used in Gokcal et al. (2009) correlation in Table 2

Curvature of the interface expressed in terms of radii of the curvature of the bubble surface, $K=\left[\frac{1}{r_{1}}+\frac{1}{r_{2}}\right]$ and $r_{1}$ and $r_{2}$ are the local principal radii of curvature at the bubble surface as indicated by Mao and Dukler (1990) (m).

Dimensionless curvature of the interface (-)

Pipe length (m)

Length of the Taylor bubble (m)

Pressure $(\mathrm{Pa})$

Dimensionless pressure (-)

Dimensionless pressure for the near-horizontal case $\left(70^{\circ}<\theta<90^{\circ}\right)$ $(-)$

Dimensionless pressure for horizontal pipe case $\left(\theta=90^{\circ}\right)(-)$

Correction term in Weber et al. (1986) correlation in Table 2

Taylor bubble radius (m)

Time (s)

Dimensionless time (-)

Dimensionless time for the near-horizontal case $\left(70^{\circ}<\theta<90^{\circ}\right)(-)$

Velocity in $\mathrm{x}$-direction $\left(\mathrm{m} \mathrm{s}^{-1}\right)$

Dimensionless velocity component in x-direction (-)

Dimensionless velocity component in $\mathrm{x}$-direction for the nearhorizontal case $\left(70^{\circ}<\theta<90^{\circ}\right)(-)$

Velocity in y-direction $\left(\mathrm{m} \mathrm{s}^{-1}\right)$ 


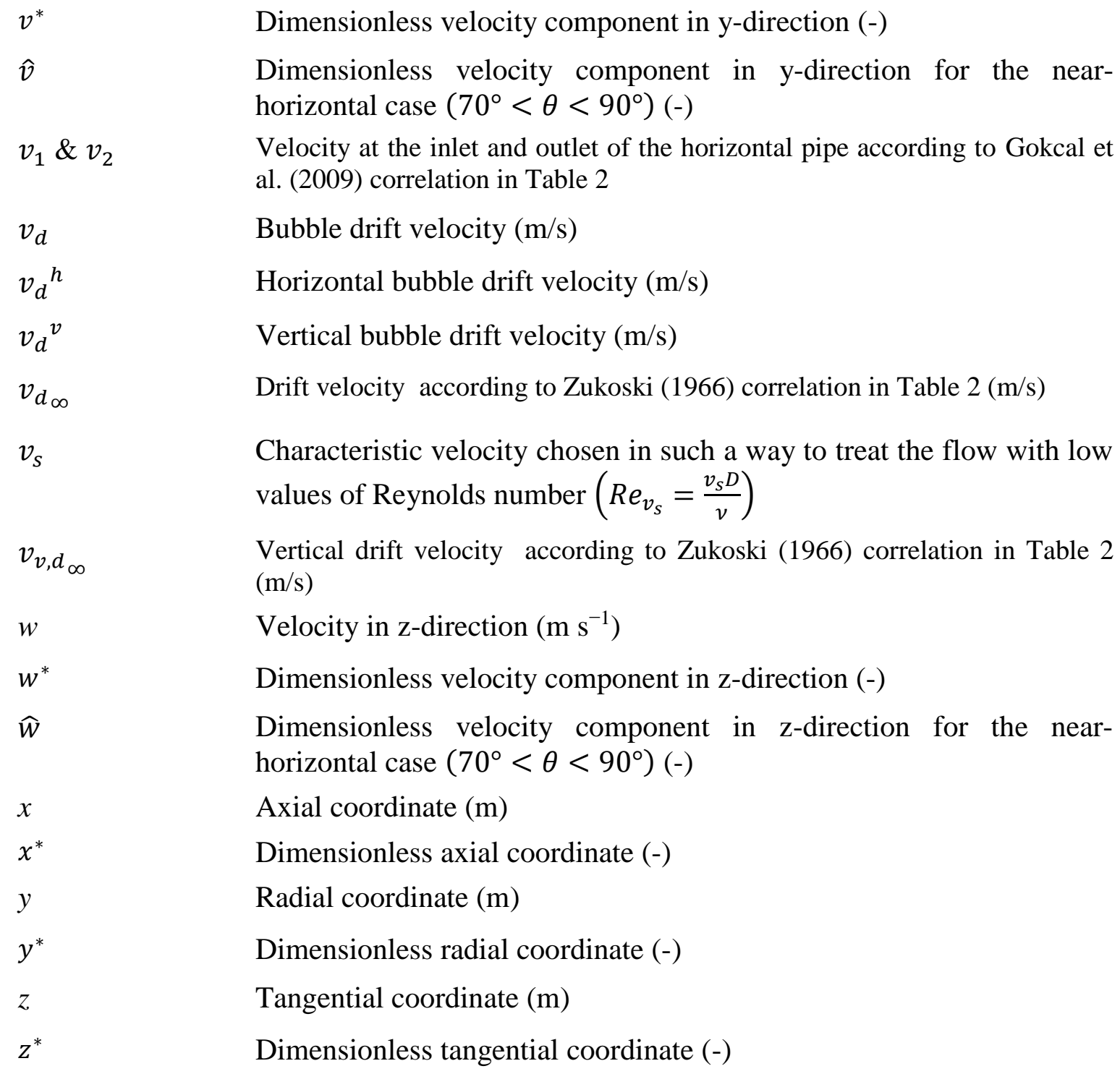




$\begin{array}{ll}\sigma_{z} & \text { Surface tension in z-direction }\left(\mathrm{N} \mathrm{m}^{-1}\right) \\ \sigma_{z}^{*} & \text { Dimensionless surface tension in } \mathrm{z} \text {-direction (-) } \\ v & \text { Kinematic viscosity }\left(\mathrm{m}^{2} \mathrm{~s}^{-1}\right)\end{array}$

\section{Dimensionless groups}

$B$

Bond number, $B o=\frac{\left(\rho_{L}-\rho_{G}\right)}{\rho_{L}} g D^{2}$

Eo

Eötvös number, $E o=\frac{g \rho_{L} D^{2}}{\sigma}$

$F r_{v_{d}}$

Froude number, $F r_{v_{d}}=\frac{v_{d}}{\sqrt{g D}}$

$F r_{v_{d}}{ }^{h}$

$F r_{v_{d}}{ }^{v}$

$G a_{R}$

$\left(\frac{L_{T B}}{D}\right)_{i}$

$\left(\frac{L_{T B}}{D}\right)_{p}$

Horizontal Froude number, $F r_{v_{d}}{ }^{h}=\frac{v_{d}{ }^{h}}{\sqrt{g D}}$

Vertical Froude number, $F r_{v_{d}}{ }^{v}=\frac{v_{d}{ }^{v}}{\sqrt{g D}}$

Reduced Galilei number, $G a_{R}=\left[\left(\frac{g L_{T B}{ }^{3}}{v_{\mathrm{L}}{ }^{2}}\right)\left(\frac{D}{2 L_{T B}}\right)^{2}\right]$

initial Taylor bubble length to pipe diameter ratio which is an input parameter prior to simulation and is fixed to 2 in all simulations

Predicted Taylor bubble length to pipe diameter ratio

$M$

Morton number, $M=\frac{\Delta \rho g \mu_{L}^{4}}{\rho_{L}^{2} \sigma^{3}}$

$N_{f}$

$R e_{v_{d}}$

Inverse viscosity number, $N_{f}=\rho_{L}\left(g D^{3}\right)^{0.5} / \mu_{L}$

Reynolds number based on Taylor bubble drift velocity, $R e_{v_{d}}=$ $\frac{\rho_{L} v_{d} D}{\mu_{L}}$

$R e_{v_{s}}$

Reynolds number based on characteristic velocity $\left(v_{s}\right), R e_{v_{s}}=\frac{v_{s} D}{v}$

\section{List of acronyms}

$\begin{array}{ll}\text { CFD } & \text { Computational fluid dynamics } \\ \text { FRF } & \text { Fixed frame of reference } \\ \text { VOF } & \text { Volume-of-fluid }\end{array}$

\section{Introduction}

Multiphase flows occur in an extensive range of applications including natural processes, chemical processes, nuclear systems and petroleum industries. The petroleum industry is considered one of the most important applications of multiphase flow; as it exists in different processes/stages such as oil processing, oil and gas transport in pipelines, and sloshing in offshore separator devices. 
Whereas the two-phase flow is classified according to the distribution of different phases building up the flow field, which known as the "flow regime/pattern". These flow patterns include bubbly, slug, plug, annular and dispersed flow. For instance, the slug flow is one of the common and complex patterns encountered in the petroleum industry. In offshore oil and gas systems, slugging problems may include flooding of downstream processing facilities, severe pipe corrosion, structural instability of pipeline; in addition to further induction of the reservoir flow oscillations, and a poor reservoir management.

Normal terrain variations are commonly observed in pipelines, consisting almost of entirely uphill and downhill inclined sections that are formed due to the land's topology. In horizontal and vertical pipes, the two-phase slug flow is considered a complex flow phenomenon. Clearly, the pipe inclination adds more complexity to the flow nature by adding another dimension to the flow in horizontal and vertical slug flows (Hernandez-Perez et al. (2010)).

The motion of bubbles in inclined pipes can affect the operation of various processes that are mainly reflected in the petroleum industry. Accordingly, the prediction of the characteristics of inclined bubble motion is essential for the successful operation, simulation and optimization of any industrial applications encountering such flow.

In literature, a substantial amount of research has been conducted so as to understand the complex principles of Taylor bubble drifting in inclined pipes with stagnant liquid. Most of these approaches are mainly experimental and/or analytical. In early stages, scientific researches were mainly focused on the motion of Taylor bubbles through liquids in inclined pipes. The works of Zukoski (1966); Bonnecaze et al. (1971); Maneri and Zuber (1974); Spedding and Nguyen (1978); Bendiksen (1984); Weber et al. (1986); Hasan and Kabir (1988); Alves et al. (1993); Abdul-Majeed (2000); Petalas and Aziz (2000), and others, have reached essential remarks on the complex motion of Taylor bubbles in inclined pipes.

Generally, Taylor bubble drift velocity in inclined pipes follows the known by "peculiar trend" with the inclination angle. This can be defined as the inclination angle increase from the horizontal position $(\theta=0)$ Froude number increases reaching a maximum value, and then decreases. Bonnecaze et al. (1971) clarified the maximum in the drift velocity detected at 30$50^{\circ}$ qualitatively in terms of the gravitational potential that drives the liquid velocity along the curved surface at the bubble nose, which initially increases and then declines with the inclination angle variation from the vertical to the horizontal positions (Wang et al. (2014)). 
More recently, advances in the experimental techniques enabled detailed investigation of the hydrodynamic characteristics of the two-phase slug flow in inclined pipes as in the studies of Roitberg et al. (2008); Shuhua et al. (2009); Hua et al. (2009) and Zhang et al. (2009). The wire-mesh sensor provides qualitative measurements of the cross-sectional void fraction distribution, statistical analysis of characteristic parameters of downward slug flow with flowing liquid, such as bubble and liquid slug length distributions, as well as the ensembleaveraged shapes of the bubble nose, liquid film and bubble tail. In addition, high-speed motion analyser technique provides competent analysis of the effect of inclination on liquid slug and Taylor bubble velocity for Taylor bubble rising through stagnant liquid.

Furthermore, other experimental studies focused on using medium and high viscous oil in addition to exploring their effect on the main hydrodynamic feature of the Taylor bubble flow (Gokcal et al. (2009); Jeyachandra et al. (2012); Moreiras et al. (2014) and Losi and Poesio (2016)). Generally, the drift velocity increases with the increase in pipe diameter and decreases with the increase in the liquid viscosity, with a maximum drift velocity at $30-50^{\circ}$ inclination.

Lastly, Roitberg et al. (2016) experimentally investigated the effect of pipe inclination and gas and liquid flow rates on elongated bubble shape in continuous slug flow in pipes with an inclination angle ranging from $2-90^{\circ}$ from the horizontal position. Similarly, they developed a theoretical film drainage model to predict the extent of the upper liquid film domain by combining Taitel and Barnea's (1990) model for the lower liquid film as both models are expected to agree well with each other.

Beside these experimental and/or analytical studies, Couët and Strumolo (1987) also attempted to develop the numerical analysis for Taylor bubble flow in inclined pipes, with $2 \mathrm{D}$ analysis (based on Newton's method), in addition to determining the bubble shape and the influence of surface tension and viscosity; and the inclination angle on Froude number. The numerical results for the bubble shape and the drift velocity showed good matching when tested against the theoretical and experimental work in literature. Their main contribution is that the dependence on the inclination angle decreases as the viscous effect increases.

Computational fluid dynamics (CFD) has also proven to be a powerful, practical tool for the analysis and simulation of the hydrodynamic characteristics of the slug flow in inclined pipes. Generally, in literature, there are no extensive CFD studies on Taylor bubbles in inclined pipes since they are computationally expensive. The assumption of the axisymmetry, as in vertical pipes, can no longer be applied. Owing to the fact that real bubbles have curvature in the 
spacing direction, a truthfully plane bubble cannot be attained. Subsequently, full 3D simulations are necessary for such simulations. It is worth to mention that few number of CFD studies are available in the literature as they investigate the inclined bubble motion with flowing liquid (Hernandez Perez (2008); Mazza et al. (2010); Hernandez-Perez et al. (2010) and Pokusaev et al. (2016)).

For inclined bubble motion with stagnant liquid, Taha and Cui (2006) performed 2D axisymmetric simulations of vertical pipes with stagnant fluid and reported only one case of 3D simulations of inclined cases. Through applying this, they presented the capability of the volume-of-fluid (VOF) method in tackling the 3D complex slug flows in inclined pipes as well as providing a complete analysis of the problem.

Most of the studies are mainly focused on investigating Taylor bubble drift velocity in inclined pipes; however, some of the studies have dealt with the liquid film region and its effect on the bubble motion (Behafarid et al. (2015) and Lizarraga-García (2016)).

Lastly, the computational domain in buoyant bubble problems needs to be large enough to capture the phenomena effectively. This is necessary in order to enable the bubble to reach a steady state condition. Different approaches were also employed in the literature with the aim of minimizing computational resources. For instance, Gutiérrez et al. (2017) employed a domain optimization method and used an Arbitrary Lagrangian-Eulerian formulation to numerically solve the three dimensional Taylor bubbles drifting in stagnant liquid. The method is based on a moving mesh that follows the drift of the Taylor bubble. Numerical stability and worthy performance are achieved when applying the method and testing the three-dimensional standard rising bubble against experimental data from the literature. A full analysis of the problem was done, including examining the effect of initial Taylor bubble shape, the initial volume of the bubble, and the tube inclination on the dynamics of Taylor bubbles.

To the authors' knowledge, despite the substantial studies, which include numerical, experimental and theoretical studies, on the drift of Taylor bubble through stagnant liquid in inclined pipes, there still are not any studies investigating the problem relying on the order of magnitude analysis of the equations of motions. Hence, the aim of this study can be divided into two main folds:

1. performing a complete dimensionless analysis of the problem using both the Buckingham-Pi theorem and a dimensionless treatment followed by an order of magnitude analysis to the governing equations of motion. This covers pipe inclination 
from vertical up to horizontal positions $\left(0 \leq \theta \leq 90^{\circ}\right)$. The analysis shows the main dimensionless similarity parameters $R e_{v_{d}}, F r_{v_{d}}$, and $E o$ numbers and their relative merits or order of magnitudes. New dimensionless similarity parameters, the reduced Gallelei number $\left(G a_{R}\right)$, is also introduced to govern the bubble dynamics in the near-horizontal cases.

2. Using the guiding principle of the order of magnitude analysis, the main hydrodynamic features of drifting of an individual Taylor bubbles through stagnant Newtonian liquids in an inclined pipe are investigated. This is actually achieved by applying computational fluid dynamics (CFD) simulation using the volume-of-fluid (VOF) methodology implemented in the commercial software ANSYS Fluent. Owing to the fact that the numerical simulation of the problem is computationally expensive, the developed numerical results considerably agree and support the order of magnitude analysis.

The work is organized as follows: section 2 presents the dimensionless equations of motion, section 3 includes details about the CFD model used. Subsequently, section 4 presents the numerical results, in addition to providing a complete description of the effect of the main similarity parameters, based on the pipe inclination and on the dynamics of the motion of a single Taylor bubble through stagnant liquid in an inclined pipe. A comparison with previous works is also given in section 4.4. Finally, section 5 presents the concluding remarks.

\section{Dimensionless equations of motion}

A complete dimensionless analysis of the motion of Taylor bubble through stagnant liquid in a vertical pipe is given in Massoud et al. (2018). Following a similar approach, the problem here is presented in a dimensionless form using both the Buckingham-Pi theorem and the dimensionless treatment of the main governing equations of motion.

The dimensionless analysis of the main governing equations: the continuity and momentum equations, followed by the order of magnitude analysis in all coordinate directions and on the boundaries is illustrated in details in Appendix 1. This analysis is based on the "peculiar trend" of the drift of Taylor bubble through stagnant liquid in inclined pipe, where the drift velocity increases with the inclination angle (with respect to the vertical position) reaching a maximum value at around $30^{\circ}$ to $50^{\circ}$, and then decreases. Additionally, a special treatment is given to the near-horizontal and horizontal cases with diminishing drift velocity. 
Based on the dimensionless analysis of the governing equations followed by the order of magnitude analysis in all coordinate directions and on the boundaries, given in Appendix 1, the main results can be summarized as follows:

- For inclination angle range of $0 \leq \theta \leq 70^{\circ}$; the main physical parameters influencing the motion of Taylor bubbles are: Froude, Eötvös and Reynolds numbers with the following magnitudes that $F r_{v_{d}}$ should be of order (1), $R e_{v_{d}}$ and $E o$ both should be of $\operatorname{order}\left(\frac{L_{T B}}{D}\right)$.

- For the near-horizontal cases $\left(70^{\circ}<\theta<90^{\circ}\right)$; where the drift velocity is vanishingly small, the main physical parameters influencing the flow are the so-called reduced Galilei number $\left(G a_{R}\right)$, and Eötvös number, with the following magnitudes that $G a_{R}$ should be of order (1) and $E o$ should be of order $\left(\frac{L_{T B}}{D}\right)$, while taking into consideration that the Reynolds and Froude numbers are vanishingly small and are no longer acting as influencing parameters.

- For the horizontal pipe case, that is for $\theta=90^{\circ}$, the sole governing parameter that governs the pressure filed distribution around the stagnant deformed bubble is Eötvös number $(E o)$. The reduced Galilei number $\left(G a_{R}\right)$ ceases to be effective, whereas Reynolds and Froude numbers are no longer influencing parameters.

To support this dimensionless analysis, the Buckingham-Pi theorem is applied for inclination angle range of $0 \leq \theta \leq 70^{\circ}$. Using the physical characteristics of the flow and geometry: $\rho_{L}, \mu_{L}$, and $\mathrm{D}$, the Taylor bubble drift velocity $\left(v_{d}\right)$ can be expressed as follows:

$$
F r_{v_{d}}=\frac{v_{d}^{2}}{g D}=f\left[E o=\frac{g \rho_{L} D^{2}}{\sigma}, R e_{v_{d}}=\frac{\rho_{L} v_{d} D}{\mu_{L}}, \frac{L_{T B}}{D}\right]
$$

However, for the near-horizontal case with an inclination range of $70^{\circ}<\theta<90^{\circ}$, the surface tension $(\sigma)$ can be physically expressed as follows:

$$
\sigma=f\left[\rho_{L}, \mu_{L}, g, L_{T B}, D\right]
$$

Using the same characteristics and applying the Buckingham-Pi theorem, three dimensionless groups can be obtained as follows:

$$
\Pi_{1}=\frac{g \rho_{L}{ }^{2} D^{3}}{\mu_{L}{ }^{2}}=\left(\frac{g L_{T B}{ }^{3}}{v_{L}{ }^{2}}\right)\left(\frac{D}{L_{T B}}\right)^{3}
$$




$$
\begin{aligned}
& \Pi_{2}=\sigma \rho_{L}{ }^{1} \mu_{L}{ }^{-2} D^{1}=\frac{\rho_{L} \sigma D}{\mu_{L}{ }^{2}} \\
& \Pi_{3}=L_{T B} D^{-1}=\frac{L_{T B}}{D}
\end{aligned}
$$

From relations (3) and (4), it can be shown that:

$$
\frac{\Pi_{1}}{\Pi_{2}}=\left(\frac{g \rho_{L}{ }^{2} D^{3}}{\mu_{L}{ }^{2}}\right) \times\left(\frac{\mu_{L}^{2}}{\rho_{L} \sigma D}\right)=\frac{g \rho_{L} D^{2}}{\sigma}=E o
$$

Additionally, from relations (3) and (5), it can be shown that:

$$
\Pi_{1} \Pi_{3}=\left(\frac{g L_{T B}{ }^{3}}{v_{L}{ }^{2}}\right)\left(\frac{D}{L_{T B}}\right)^{3} \times\left(\frac{L_{T B}}{D}\right)=\left(\frac{g L_{T B}{ }^{3}}{v_{L}{ }^{2}}\right)\left(\frac{D}{L_{T B}}\right)^{2}=G a_{R}
$$

Hence, it can be concluded that the sole governing parameters for the near-horizontal case are the reduced Galilei number $\left(G a_{R}\right)$, Eötvös number $(E o)$; and $\frac{L_{T B}}{D}$.

Lastly, for the horizontal case, that is for $\theta=90^{\circ}$, the surface tension $(\sigma)$ can be expressed as follows:

$$
\sigma=f\left[\rho_{L}, g, L_{T B}, D\right]
$$

Similarly, by applying the Buckingham-Pi theorem, two dimensionless groups can be obtained as follows:

$$
\begin{aligned}
& \Pi_{1}=\sigma \rho_{L}{ }^{-1} g^{-1} D^{-2}=\frac{\sigma}{\rho_{L} g D^{2}}=\frac{1}{E o} \\
& \Pi_{2}=L_{T B} D^{-1}=\frac{L_{T B}}{D}
\end{aligned}
$$

Relations (9) and (10) emphasise that the main similarity parameters that govern the pressure field distribution around the stagnant deformed bubble are Eötvös number $(E o)$ and $\frac{L_{T B}}{D}$.

In conclusion, the dimensionless analysis using both methods; the Buckingham-Pi theorem and the dimensionless treatment of the governing equations followed by the order of magnitude analysis, shows that the main governing parameters that influence the drift velocity of Taylor bubbles through stagnant liquid in inclined pipe are Froude, Eötvös and Reynolds numbers, similar to the vertical pipe case given in Massoud et al. (2018). Nonetheless, a special treatment of the near-horizontal cases $\left(70^{\circ}<\theta<90^{\circ}\right)$ emphasizes that Reynolds and Froude numbers 
are no longer the main influencing parameters as the so-called reduced Galilei number $\left(G a_{R}\right)$, and Eötvös number are currently the main physical parameters influencing the flow. Lastly, for the horizontal pipe case, the only similarity paramter governing the stagnant deformed bubble is Eötvös number $(E o)$.

Regarding the independence of the cylindrical bubbles' velocity of the Taylor bubble length provided that the bubble is long enough (more than 1.5D), the effect of ratio of bubble size to the pipe diameter $\left(L_{T B} / D\right)$ is considerably negligible throughout this study (White and Beardmore (1962) ; Nicklin et al. (1962) ; Zukoski (1966); Mao and Dukler (1990) and Polonsky et al. (1999)). Accordingly, to support the developed logical approach to the problem, the main dynamic features of an individual Taylor bubble drifting through stagnant Newtonian liquids in an inclined pipe are investigated by applying a computational fluid dynamics (CFD) simulation.

\section{CFD Model development}

The flow domain is created and solved using the volume-of-fluid (VOF) methodology implemented in the computational fluid dynamics software package, ANSYS Fluent (Release 16.0). While details about the fluid properties and the numerical method used in the present work can be found in Massoud et al. (2018). Figure 1 displays a schematic representation of the computational domain for the inclined pipe configuration. Once the pipe is inclined with respect to the vertical position, the symmetry of the vertical pipe configuration is lost. Thus, the assumption of the flow being axisymmetric can no longer be applied. Nevertheless, the flow can be simplified as a plane of symmetry that crosses the pipe's diameter line with a normal orthogonal to the gravitational acceleration.

As indicated in Figure 1, the solution domain is a cylindrical domain with diameter $(D)$ and length $(L)$ and the pipe's inclination is configured by setting the angle $(\theta)$ between the pipe's axis and the gravity vector with regard to the vertical case. Moreover, the length of the computational domain is kept at 10 to $11 \mathrm{D}$ to ensure that the solution is affected neither by the bottom nor the top of the pipe. However, all simulations for the inclined pipe cases are done using a fixed reference frames (FRF) system. The initial bubble shape is composed of a cylinder connected to a hemisphere with the same radius giving an overall bullet shape of Taylor bubble. The length and radius of the Taylor bubble are given by $L_{T B}$, and $R_{T B}$, respectively.

A test was also done to investigate the effect of the distance from the pipe's bottom, given by $h_{B}$, with three different values of $h_{B}=1 \mathrm{D}, 1.5 \mathrm{D}$ and $2 \mathrm{D}$. It was concluded that the three different 

Additionally, all the velocity components are set at zero. For the gas-liquid interface, the same kinematic and dynamic boundary conditions are applied.

The initial bubble shape is a bullet shaped with a cylinder connected to a hemisphere with the same radius. While the volume of the gas phase is specified using the patch option in Fluent. The initial ratio of Taylor bubble length to pipe diameter $\left(L_{T B} / D\right)_{i}$ is an input parameter prior to simulation and is fixed to 2 in all simulations. In addition, it should be highlighted that different Taylor bubble shapes, sizes and locations were tested and they accurately gave the same solutions. Although the only difference was in the convergence process, the selected Taylor bubble initial shape and location showed a well converged solution.

As regarding the spatial domain, Figure 1 shows the block-structured O-Grid hexahedral used which is based on the work Hernandez-Perez et al. (2011) and Ambrose (2015). This mesh has proven to accurately predict the flow behaviour in liquid film regions and is also suitable for the modeling of slug flow applications, and accordingly the drift of Taylor bubbles in pipes.

Finally, the initial shape is simulated while tracking the bubble velocity until a steady terminal developed bubble shape with a converged terminal constant bubble drift velocity $\left(v_{d}\right)$ is reached. Based on the studied cases, the size of the mesh is selected and refined until the terminal velocity stabilizes and converges. Generally, the number of cells ranges from 1 million up to 10 million cells. Additionally, details regarding the solution strategy and the convergence criterion used in the simulations, thoroughly presented in Massoud et al. (2018).

\section{Results and discussions}

In the current section, the main findings of this work are discussed. A validation study of single Taylor bubble drifting through stagnant liquid is given in Massoud et al. (2016\&2018). Based on the analysis given in section 2 , the results are then divided into three main sections; section 4.1 investigates the dynamics of Taylor bubble in an inclined pipe of an inclination range of $0^{\circ} \leq \theta \leq 70^{\circ}$, exploring the effect of the sole dimensionless parameters: Froude, Eötvös and 
Reynolds numbers on the dynamics of Taylor bubbles. Section 4.2 covers the near-horizontal section for the pipe inclination range of $70^{\circ}<\theta<90^{\circ}$, with the reduced Galilei number and Eötvös number acting as the main influencing parameters. Furthermore, Section 4.3 examines the horizontal pipe section with $\theta=90^{\circ}$ with Eötvös number being the only influencing parameter. In each section, the dynamics of the Taylor bubbles include the final shape of the developed Taylor bubble, the flow field around it (streamlines), and the Taylor bubble terminal drift velocity. Finally, section 4.4 provides a comparison with the previous experimental work where the present simulation results are compared against well-known correlations and experimental work available in the literature.

\subsection{Inclination range of $0 \leq \theta \leq 70^{\circ}$}

In this section, the effect of the main physical parameters influencing the motion of Taylor bubbles: Froude, Eötvös and Reynolds numbers, on the dynamics of Taylor bubbles, are investigated. Details about the working fluid properties and their corresponding dimensionless numbers are also given in Massoud et al. (2018). The pipe inclination covers the following angles: $0^{\circ}, 15^{\circ}, 30^{\circ}, 45^{\circ}, 60^{\circ}$ and $70^{\circ}$, Eötvös number has values of 20 and 66, and Reynolds number has average values of 15,25 and 95 .

\subsubsection{Effect of inclination angle}

To explore the effect of inclination angle on the terminal Taylor bubble shape as well as exploring further details about the streamlines and vorticity field, a selected case of the following parameters; $E o=66$ and $R e_{v_{d}} \cong 15$, is presented. It can be noted from Figure 2 that the developed Taylor bubble length increases with the inclination angle. For each inclination angle the developed bubble length is divided into three sections (section A-A to section $\mathrm{C}-\mathrm{C}$ ). For each section, the streamlines and vorticity field (Y-component) in the $\mathrm{XZ}$ plane are presented in Figure 2. The vorticity slightly increases with the inclination angle. Through considering the highest section (section A-A), the flow shows similar configurations for the all inclination angles. Nonetheless, the instability of the bubble rear is shown by the disturbed flow as demonstrates in section $\mathrm{C}-\mathrm{C}$, where secondary vortices appear at the perimeter of the pipe in almost all inclination angles.

In addition, the effect of pipe inclination on the time evolution of the Froude number $\left(F r_{v_{d}}\right)$ is given in Figure 3, where the fastest convergence in the time evolution of $F r_{v_{d}}$ is established by the vertical pipe case. A similar behavior of $F r_{v_{d}}$ evolution is also noticed for angles $15^{\circ}$ to 
$45^{\circ}$. However, for larger inclination angles, a different evolution is noticed especially with an inclination angle of $70^{\circ}$ where longer simulation time is essential for establishing constant terminal $F r_{v_{d}}$.

\subsubsection{Effect of Reynolds number}

In order to explore the consequences of Reynolds number on the dynamics of Taylor bubble, two selected cases with fixed Eötvös number of 66 but with different Reynolds number corresponding to approximate values of 15 and 95 are simulated. Moreover, Figure 4 illustrates the effect of $R e_{v_{d}}$ on the final shape of the developed Taylor bubble, the flow field, and the terminal Froude number.

At low values of Reynolds number, the high viscous forces increase the elongation of the terminal developed Taylor bubble with the most extended Taylor bubble being at the vertical case, as indicated in Figure 4(a). Generally, for the vertical case, a pair of elongated counterrotating vortices are noticed in the bubble wake. As the pipe inclination increases, this configuration is lost. A vortex close to the wall starts to appear with the inclination angle which opens up with the increase in inclination angle. Another vortex at the far side of the wall originates at a higher inclination.

The increase in Reynolds number affects the average velocity of the flow for all angles, and the vortex development as well. It also affects the bubble rear shape by increasing its concavity as shown in Figure 5. As for the developed bubble wake, it can be noticed that the Reynolds number has a significant effect on the intensity and scale of the wake.

The effect of Reynolds number on the terminal Froude number is given in Figure 4(c). For low Reynolds number values, the viscous forces of liquid phase surrounding the bubbles are high enough to weaken the drift of the Taylor bubble, and the resistance for the bubble to penetrate the stagnant liquid is high and is capable of reducing its drift velocity. Generally, for a fixed Reynolds number, the bubble velocity increases with the inclination angle reaching its maximum value at $45^{\circ}$. Obviously, further increase in the inclination angle reduces the terminal bubble velocity. This behaviour was clearly examined by Maneri and Zuber (1974) and Spedding and Nguyen (1978): as the angle of inclination increases, the buoyancy forces increase which subsequently increases the bubble velocity. A resistive force opposes this force from the draining of the liquid past the rising bubble. The results agree with the conclusions of Maneri and Zuber (1974) where the maximum Froude value occurs at an angle (45 ${ }^{\circ}$ based on 
current cases) at which the transition from a blunt-nosed bubble-double falling film system to a streamlined bubble with almost one falling film system occurs. Hence, it can be concluded that at lower Reynolds number, the blunter bubble nose is observed to result in a reduction in the bubble drift velocity.

\subsubsection{Effect of Eötvös number}

The effect of buoyancy and surface tension forces on the motion of Taylor bubbles in inclined pipes can be examined through considering the impact of Eötvös number. Two cases are simulated with Eo values of 20 and 66, and Reynolds number with an average value of 25.

Figure 6 shows the effect of Eo number on the final shape of the developed Taylor bubble, the flow field, and the terminal Froude number. Similar to the vertical pipe case, the surface tension is high at low values of Eo, that avoid any possible distortion at the interface. In Figure 6(a), the bubble starts with prolate spheroidal nose and oblate spheroid bottom at the vertical position. This shape is considerably maintained with most of the inclination angles with an increase in the bubble's length as a result of the pipe's inclination.

As indicated in Figure 7, the increase in Eo number, alters the shape of the bubble tail from convex into a concave structure, which reflects into wider wakes. It should be pointed out that for $E o \geq 100$, highly disturbed bubble geometry is noticed for various inclination angles with an un-converged bubble velocity, especially for an inclination range of $15^{\circ} \leq \theta \leq 45^{\circ}$. Thus, the results suggest that for $E o \geq 100$, the effect of surface tension results in deformation at gas-liquid interface, and hence it loses its structure and a strong breaking up concept is noticed. Also, for $E o \leq 10$, no Taylor bubble exists especially for large inclination angles.

The effect of Eötvös number on the terminal Froude number is given in Figure 6(c). For fixed $E o=20$, the terminal velocity increases with the inclination angle with maximum values of $45^{\circ}$. Whereas, the increase in $E O$ values results in an increase in the gravitational potential that enables the bubble to drift through the stagnant liquid. Both the drift velocity and the gravitational potential reach a maximum value of $45^{\circ}$ for $E o=20$, and $60^{\circ}$ for $E o=66$. Due to increase in $E o$ values, there is a tendency of change in maximum Froude number values with larger inclination angle. This conclusion agrees with the conclusions of Maneri and Zuber (1974); Weber et al. (1986); Shosho and Ryan (2001) and Jeyachandra et al. (2012).

In conclusion, it can be deduced that both $E o$ and $R e_{v_{d}}$ significantly control the dynamics of the motion of bubbles in inclined pipes. Based on the simulation cases, the results suggest that 
a higher Eo range is essential to maintain stable Taylor bubble formation in inclined pipe when compared with the vertical cases. Adding to that, the instability in the gas-liquid interface is also noticed for $E o \geq 100$. The drift velocity followed the "peculiar trend" with the inclination angle for $R e_{v_{d}}$ values investigated. However, the maximum Froude values occur at higher angles with the increment in Eo values.

\subsection{Inclination range of $70^{\circ}<\theta<90^{\circ}$}

Special treatment of the near-horizontal cases, covering the inclination range of $70^{\circ}<\theta<$ $90^{\circ}$, is given in section 2. As explained earlier, the dynamics of the Taylor bubble in this inclination range is governed mainly by; the reduced Galilei number $\left(G a_{R}\right)$, and Eötvös number $(E o)$. To support the results of this dimensionless analysis, selected cases with inclination angles of $75^{\circ}$ and $85^{\circ}$, Eötvös number values of 66 and 100, and Galilei number of 15 and 200 are simulated. It should be pointed out that significant elongation in Taylor bubble length has been noticed for this inclination range with an infinite Taylor bubble length established in a horizontal pipe. Thus, a longer domain is essential for these cases with pipe length fixed to $14 \mathrm{D}$.

\subsubsection{Effect of reduced Galileo number}

The Taylor bubble drift velocity significantly decreases for the pipe inclination range of $70^{\circ}<$ $\theta<90^{\circ}$, until the bubble stops moving in the horizontal orientation. Based on that, the reduced Galilei number $\left(G a_{R}\right)$, which represents the ratio between the gravitational and the viscous forces based on characteristic velocity $\left(v_{s}\right)$ that treats low Reynolds values, is suggested to govern the bubble dynamics. To explore the effect of $G a_{R}$ on the dynamics of Taylor bubbles, two cases with fixed $E o$ values of 66, and $G a_{R}$ values of 15 and 200 are simulated. Additionally, Figure 8 shows the terminal bubble shape and the streamlines in the XY plane for $G a_{R}$ values of 15 and 200. Long, streamlined bubbles are also noticed to develop for this inclination range for all $G a_{R}$ values. The bubble occupies the upper part of the pipe with a thinly formed upper liquid film. With regards to cases 1 and 2 in Table 1, it can be noted that the increase in $G a_{R}$ values increases the bubble motion, but generally, all cases has low $F r_{v_{d}}$ values. Reduction in the developed Taylor bubble length is noticed with larger $G a_{R}$. 


\subsubsection{Effect of Eötvös number}

The effect of Eötvös number on the dynamics of Taylor bubbles in near-horizontal cases is investigated for cases 3 and 4 in Table 1, with a fixed $G a_{R}$ value of 200, and Eötvös number values of 66 and 100. Figure 9 shows the bubble shape profile for cases 3 and 4 in Table 1. It can be noticed that the increase in Eo values reduces the sizes of the developed bubble as well as enhances its elongation. The higher bouyancy forces resulting from higher Eo values, leads to a faster bubble motion, which results in higher $F r_{v_{d}}$ values as indicated in Table 1.

It can be concluded that the bubble motion in this inclination range is considerably low in comparison to other inclination angles. Clearly, the effect of the reduced Galilei number is mainly reflected in the bubble's motion; however, the bubble's length enhancement does not significantly change with $G a_{R}$. Eo effect is still vital similar to the other inclination angles. The effect of $E o$ can be seen in the developed bubble length and size, bubble shape, as well as $F r_{v_{d}}$ values. Additionally, it is worth mentioning that accordong to the simulated cases, larger values of $E o(E o \geq 40)$ are essential for Taylor bubble to exist within this inclination range.

The governing equations, given in section 2 , shows that when the pipe inclination approaches the horizontal position, the problem can be approximated using the $2 \mathrm{D}$-transient analysis. To support this conclusion, for inclination angles of $85^{\circ}, 87^{\circ}$ and $89^{\circ}$, the $3 \mathrm{D}$ transient model is tested against the 2D-transient model, as indicated in Figure 10 until Figure 12. It is worth mentioning that $E o$ is of order $o\left(\frac{L_{T B}}{D}\right)$ for all these angles of inclination. However, as the inclination angle moves towards the horizontal position, the developed bubble's length becomes significantly large. Hence, for instance, for an angle of $85^{\circ}$ Eo should be $\geq 40$ to ensure a stable formation of the Taylor bubble, while larger angles, such as $89^{\circ}$, Eo should be $\geq 100$. It can be generally deduced that the 2D-transient model can offer an approximate solution for Taylor bubble shapes for angles of $85^{\circ}$ to $87^{\circ}$. However, for larger angles $\left(\theta=89^{\circ}\right)$ the solution can be more simplified by using the $2 \mathrm{D}$-steady model.

\subsection{Inclination range of $\theta=90^{\circ}$}

As the pipe inclination approaches the horizontal orientation, the bubble velocity diminishes until it becomes zero at the horizontal position. In literature, there are various discussions describing the bubble behaviour in horizontal pipes. For instance, based on the fact that gravity does not act in horizontal direction, Wallis (1969) and Dukler and Hubbard (1975) state that there is no bubble motion in a horizontal system. Whereas, others suggest that the bubble 
propagates in the horizontal pipe due to the pressure difference between the top and the bottom of the bubble nose (Weber (1981) and Bendiksen (1984)), which is not the case for the present zero pressure gradient analysis.

In the literature, most of the experimental work measures the bubble velocity at different inclinations including the horizontal position either by measuring the liquid drainage velocity or by creating an axial pressure gradient, by opening the pipe's top, that enhances the bubble motion (Spedding and Nguyen (1978); Weber et al. (1986); Gokcal et al. (2009); and Jeyachandra et al. (2012)). However, the main argument is that Froude number's estimation should not be based on either of these cases. Hence, the present work is based on the real case with zero axial pressure gradient analysis in a horizontal pipe.

In the horizontal pipe case, the developed bubble eventually has an infinite length. The problem can be simplified using a 2D-steady solution, where the only governing parameter is Eo which governs the static pressure distribution around the stagnant deformed bubble. Whereas Galilei number ceases to be effective, and Reynolds and Froude numbers are no longer effective parameters. A selected case with a high Eo value of 300 is simulated using the 3D-transient model, 3D-steady model and the simplified 2D-steady model. As indicated in Figure 13, the 2D-steady model successfully predicts the developed Taylor bubble shape. Hence, by using this model, the effect of $E o$ on the predicted Taylor bubble can be investigated using $E o$ values of; 300, 500, 700 and 1000. It can be concluded from Figure 14, that Eo affects the size of the developed Taylor bubble, where the increase in Eo leads to a more flattened bubble.

\subsection{Comparison with previous works}

In literature, most of the research focuses on exploring the bubble drift velocity, expressed in terms of Froude number, with the inclination angle. Additionlly, Table 2 gives a summary of the main correlations proposed in the literature for bubble drift velocity with their limitations. One of the reliable experimental work is proposed by Shosho and Ryan (2001) who studied the motion of long bubbles in inclined tubes for Newtonian and non-Newtonian fluids covering a wide range of dimensionless groups. In Table 3, two cases based on the work of Shosho and Ryan (2001) are also selected for the sake of comparison of the present simulation against the experimental data. The working fluids for these cases are air and sucrose solutions with different concentrations. 
Figure 15 shows the developed Taylor bubble shape and the terminal Froude number $\left(F r_{v_{d}}\right)$ compared against the experimental results of Shosho and Ryan (2001) for case 1 given in Table 3. For the Taylor bubble shape, the bubble loses it symmetry once the pipe is inclined with respect to the vertical position. In fact, the pipe diameter is reduced with the inclination angle which leads to an elongation of the bubble length. While, the upper liquid film thickness (the upper distance between the bubble and the wall) is reduced with the increase in the inclination angle as the bubble nose is shifted from the center of the pipe, at the vertical position, towards the wall.

Additionally, it should be pointed out that for inclination angle $\theta=75^{\circ}$ as in Figure 15 , a mesh check is done to ensure the convergence of the terminal drift velocity and stability in terminal bubble shape. However, increasing the cells' number requires large computational resources, and subsequently, the mesh selected for such case ( $\cong 7$ million cells) is considerably suitable to obtain a converged Taylor bubble velocity. Nevertheless, the instability in the developed Taylor bubble shape is caused by large values of reduced Galilei number $\left(G a_{R}=9311\right)$ which is inconvenient to have for these inclination ranges based on the discussion provided in section 2.

For the Taylor bubble drift velocity, the bubble drift velocity follows the expected "peculiar trend" concerning the inclination angle. This "peculiar trend" is noticed by most of the researchers with different conclusions on the angle at which maximum Froude number $\left(F r_{d}\right)$ occurs. However, the general conclusion is that the Froude number $\left(F r_{v_{d}}\right)$ reaches its maximum value at an inclination angle; $30^{\circ}<\theta<60^{\circ}$ as a result of the opposing effects of buoyancy and the drag coefficient. For inclination angles approaching the horizontal position, the bubble terminal velocity drops expressively to approach a zero value.

Some of the main correlations given in Table 2 are presented in Figure 15 as to compare the present simulation results. Obviously, most of the correlations follow the expected "peculiar" trend with respect to the inclination angle. However, the main difference between the correlations is noticed in the near-horizontal cases where some consider no bubble motion in the horizontal position and others suggest that there is bubble motion in such position. The limitation of each correlation is given in Table 2. One of the reliable correlations is suggested by Lizarraga-García (2016) as it can predict the Froude number for wide range of Eo with almost no limitations. 
Concerning Figure 15 and Figure 16, it can be concluded that the simulation results show a qualitative agreement with the experimental results of Shosho and Ryan (2001) and the correlation proposed by Lizarraga-García (2016) for the two cases provided in Table 3.

In conclusion, based on the main correlations proposed in the literature, it can be noticed that improvements in predicting Taylor bubble drift velocity are essential. Moreover, the numerical results showed a reasonable agreement with the experimental data and the correlations of bubble drift velocity.

\section{Conclusion}

The main purpose of this work is to perform a complete dimensionless analysis of single Taylor bubble drifting through stagnant Newtonian liquid in an inclined pipe, followed by an order of magnitude analysis of the equations of motion. The main conclusion reached is that different governing parameters appear according to the range of pipe inclination angle. For inclination range of $0 \leq \theta \leq 70^{\circ}$; the problem is mainly governed by Froude, Eötvös and Reynolds numbers. As for near-horizontal cases $\left(70^{\circ}<\theta<90^{\circ}\right)$ the main physical parameters influencing the flow are the so-called reduced Galilei number and Eötvös number, while taking into consideration that the Reynolds and Froude numbers are vanishingly small and are no longer acting as influencing parameters. Finally, for the horizontal pipe case, the pressure field distribution around the stagnant deformed bubble is mainly governed by the Eötvös number, where the reduced Galilei number ceases to be effective similar to the Reynolds and Froude numbers.

Using the guideline of the order of magnitude analysis and the dimensionless group analysis, a CFD study investigating the hydrodynamics of single Taylor bubble drifting through stagnant Newtonian liquid in an inclined pipe is performed. Additionally, this CFD study also employs the volume-of-fluid (VOF) methodology implemented in the computational fluid dynamics software package, ANSYS Fluent (Release 16.0). Particulary, the current study is focused on the main similarity parameters, that is Froude number $\left(F r_{v_{d}}\right)$, Reynolds number $\left(R e_{v_{d}}\right)$, Eötvös number $(E o)$ and the reduced Galilei number $\left(G a_{R}\right)$. The main hydrodynamic characteristics discussed include the developed Taylor bubble shape, the flow field and the Taylor bubble drift velocity.

The main findings of this study can be summarized as follows: 
- The bubble motion follows the "peculiar trend" with the inclination angle, where the bubble velocity increases with inclination angle until it reaches a maximum value, then it starts to decrease once again. The bubble shape is significantly affected by the inclination of the pipe where elongation in Taylor bubble length with a reduction in size is established with the pipe inclination.

- Eo has a significant effect on the Taylor bubble shape and velocity. The rise in surface tension alters the shape of the bubble tail from a convex into a concave structure which is reflected into wider wakes. The increase in $E o$ values raises the gravitational potential that enables the bubble to drift through the stagnant liquid. There is also a tendency of change in maximum Froude number values with larger inclination angle due to the increase in $E o$ values.

- $R e_{v_{d}}$ shows a similar role to $E o$ where the increase in $R e_{v_{d}}$ alters the bubble shape from long slender bubble into shorter and flatter ones. At low $R e_{v_{d}}$, more blunter bubble nose is observed, and thus leads to a reduction in the bubble motion.

- $G a_{R}$ mainly affects the bubble motion with a negligible effect on the bubble shape. The increase of gravitational forces due to the increase in $G a_{R}$, increases the bubble motion, but generally, all cases has low $F r_{v_{d}}$ values.

- The bubble motion in near-horizontal cases is considerably low with significant enhancement in the Taylor bubble length. Thus, a larger domain is essential for these angles. For the main aim of reducing the computational resources, a simplified 2D model, based on the dimensionless analysis developed in this paper, is suggested to give an approximate solution to estimate the developed Taylor bubble shape.

- In addition, it can be deduced that the developed numerical results agree well with the order of magnitude analysis. For horizontal pipes, Eo shows different order of magnitude based on large length of developed Taylor bubble, where larger Eo values are essential for a stable Taylor bubble to exist $(E o \geq 200)$. By using the simplified 2D model, Eo is the only governing parameter that significantly affects the developed Taylor bubble shape and size.

- Finally, the present simulation results show a reasonable agreement when tested against the previous experimental data and the main correlations available in the literature. 
Nevertheless, it can be noticed that further improvements are still necessary for predicting the Taylor bubble drift velocity.

\section{Acknowledgements}

Results were obtained using the EPSRC funded ARCHIE-WeSt High-Performance Computer (www.archie-west.ac.uk). EPSRC grant no. EP/K000586/1.

\section{References}

ABDUL-MAJEED, G. H. 2000. Liquid slug holdup in horizontal and slightly inclined twophase slug flow. Journal of Petroleum Science and Engineering, 27, 27-32.

ALVES, I. N., SHOHAM, O. \& TAITEL, Y. 1993. Drift velocity of elongated bubbles in inclined pipes. Chemical engineering science, 48, 3063-3070.

AMBROSE, S. 2015. The rise of Taylor bubbles in vertical pipes. University of Nottingham.

BEHAFARID, F., JANSEN, K. \& PODOWSKI, M. 2015. A study on large bubble motion and liquid film in vertical pipes and inclined narrow channels. International Journal of Multiphase Flow, 75, 288-299.

BENDIKSEN, K. H. 1984. An experimental investigation of the motion of long bubbles in inclined tubes. International journal of multiphase flow, 10, 467-483.

BENDIKSEN, K. H. 1985. On the motion of long bubbles in vertical tubes. International journal of multiphase flow, 11, 797-812.

BONNECAZE, R., ERSKINE, W. \& GRESKOVICH, E. 1971. Holdup and pressure drop for two - phase slug flow in inclined pipelines. AIChE Journal, 17, 1109-1113.

COUËT, B. \& STRUMOLO, G. S. 1987. The effects of surface tension and tube inclination on a two-dimensional rising bubble. Journal of Fluid Mechanics, 184, 1-14.

DUKLER, A. E. \& HUBBARD, M. G. 1975. A model for gas-liquid slug flow in horizontal and near horizontal tubes. Industrial \& Engineering Chemistry Fundamentals, 14, 337 347.

GOKCAL, B., AL-SARKHI, A. S. \& SARICA, C. 2009. Effects of high oil viscosity on drift velocity for horizontal and upward inclined pipes. SPE Projects, Facilities \& Construction, 4, 32-40.

GUTIÉRREZ, E., BALCAZAR, N., BARTRONS, E. \& RIGOLA, J. 2017. Numerical study of Taylor bubbles rising in a stagnant liquid using a level-set/moving-mesh method. Chemical Engineering Science, 164, 158-177.

HASAN, A. R. \& KABIR, C. S. 1988. Predicting multiphase flow behavior in a deviated well. SPE Production Engineering, 3, 474-482.

HERNANDEZ-PEREZ, V., ABDULKADIR, M. \& AZZOPARDI, B. 2010. Slugging frequency correlation for inclined gas-liquid flow. World Acad Sci Eng Technol, 37, 44-51.

HERNANDEZ-PEREZ, V., ABDULKADIR, M. \& AZZOPARDI, B. 2011. Grid generation issues in the CFD modelling of two-phase flow in a pipe. The Journal of Computational Multiphase Flows, 3, 13-26. 
HERNANDEZ PEREZ, V. 2008. Gas-liquid two-phase flow in inclined pipes. University of Nottingham.

HUA, Z., SHUHUA, W. \& JING, W. 2009. Experimental study of motion of nitrogen Taylor bubbles and liquid slugs in inclined tubes. Chinese Journal of Aeronautics, 22, 349354.

JEYACHANDRA, B., GOKCAL, B., AL-SARKHI, A., SARICA, C. \& SHARMA, A. 2012. Drift-velocity closure relationships for slug two-phase high-viscosity oil flow in pipes. SPE Journal, 17, 593-601.

LIZARRAGA-GARCÍA, E. 2016. A study of Taylor bubbles in vertical and inclined slug flow using multiphase CFD with level set. Massachusetts Institute of Technology.

LOSI, G. \& POESIO, P. 2016. An experimental investigation on the effect of viscosity on bubbles moving in horizontal and slightly inclined pipes. Experimental Thermal and Fluid Science, 75, 77-88.

MANERI, C. \& ZUBER, N. 1974. An experimental study of plane bubbles rising at inclination. International Journal of Multiphase Flow, 1, 623-645.

MAO, Z.-S. \& DUKLER, A. 1990. The motion of Taylor bubbles in vertical tubes. I. A numerical simulation for the shape and rise velocity of Taylor bubbles in stagnant and flowing liquid. Journal of computational physics, 91, 132-160.

MASSOUD, E., XIAO, Q., EL-GAMAL, H. \& TEAMAH, M. 2018. Numerical study of an individual Taylor bubble rising through stagnant liquids under laminar flow regime. Ocean Engineering, 162, 117-137.

MASSOUD, E., XIAO, Q., TEAMAH, M. \& SAQR, K. 2016. Numerical Study of Hydrodynamic Characteristics of Gas-Liquid Slug Flow in Vertical Pipes. Journal of Applied Mechanical Engineering, 5.

MAZZA, R., ROSA, E. \& YOSHIZAWA, C. 2010. Analyses of liquid film models applied to horizontal and near horizontal gas-liquid slug flows. Chemical Engineering Science, 65, 3876-3892.

MOREIRAS, J., PEREYRA, E., SARICA, C. \& TORRES, C. F. 2014. Unified drift velocity closure relationship for large bubbles rising in stagnant viscous fluids in pipes. Journal of Petroleum Science and Engineering, 124, 359-366.

NICKLIN, D., WILKES, J. \& DAVIDSON, J. 1962. Two-phase flow in vertical tubes. Trans. Inst. Chem. Eng, 40, 61-68.

POKUSAEV, B., NEKRASOV, D. \& KHRAMTSOV, D. 2016. Modeling slug flow and mass transfer in inclined pipes. Theoretical Foundations of Chemical Engineering, 50, $237-$ 241.

POLONSKY, S., SHEMER, L. \& BARNEA, D. 1999. The relation between the Taylor bubble motion and the velocity field ahead of it. International Journal of Multiphase Flow, 25, 957-975.

ROITBERG, E., BARNEA, D. \& SHEMER, L. 2016. Elongated bubble shape in inclined airwater slug flow. International Journal of Multiphase Flow, 85, 76-85.

ROITBERG, E., SHEMER, L. \& BARNEA, D. 2008. Hydrodynamic characteristics of gasliquid slug flow in a downward inclined pipe. Chemical Engineering Science, 63, 36053613. 
SHOSHO, C. E. \& RYAN, M. E. 2001. An experimental study of the motion of long bubbles in inclined tubes. Chemical engineering science, 56, 2191-2204.

SHUHUA, W., ZHANG, H. \& JING, W. 2009. Cryogenic liquid slug and Taylor bubble length distributions in an inclined tube. Chinese Journal of Chemical Engineering, 17, 20-26.

SPEDDING, P. \& NGUYEN, V. T. 1978. Bubble rise and liquid content in horizontal and inclined tubes. Chemical Engineering Science, 33, 987-994.

TAHA, T. \& CUI, Z. 2006. CFD modelling of slug flow in vertical tubes. Chemical Engineering Science, 61, 676-687.

TAITEL, Y. \& BARNEA, D. 1990. A consistent approach for calculating pressure drop in inclined slug flow. Chemical engineering science, 45, 1199-1206.

WALLIS, G. B. 1969. One-dimensional two-phase flow.

WANG, Y., YAN, C., SUN, L., YAN, C. \& TIAN, Q. 2014. Characteristics of slug flow in a narrow rectangular channel under inclined conditions. Progress in Nuclear Energy, 76, 24-35.

WEBER, M., ALARIE, A. \& RYAN, M. 1986. Velocities of extended bubbles in inclined tubes. Chemical engineering science, 41, 2235-2240.

WEBER, M. E. 1981. Drift in intermittent two - phase flow in horizontal pipes. The Canadian Journal of Chemical Engineering, 59, 398-399.

WHITE, E. \& BEARDMORE, R. 1962. The velocity of rise of single cylindrical air bubbles through liquids contained in vertical tubes. Chemical Engineering Science, 17, 351361.

ZHANG, H., WANG, S. \& WANG, J. 2009. Experimental study on boiling flow of liquid nitrogen in inclined tubes-Velocities and length distributions of Taylor bubbles. Journal of the Taiwan Institute of Chemical Engineers, 40, 431-438.

ZUKOSKI, E. 1966. Influence of viscosity, surface tension, and inclination angle on motion of long bubbles in closed tubes. Journal of Fluid Mechanics, 25, 821-837.

\section{List of Tables}

Table 1- Simulation cases used to explore the effect of both reduced Galilei number GaR and Eötvös number Eo on dynamics of Taylor bubble for inclination angle range of $70^{\circ}<\theta<90^{\circ}$, with their corresponding results.

Table 2- Summary of the main correlations proposed in the literature for the rising velocity of a Taylor bubble in inclined pipes with stagnant liquid.

Table 3- Test matrix for simulations of drift of Taylor bubble through stagnant liquid in inclined pipes based on the experimental work of Shosho and Ryan (2001). 


\section{List of Figures}

Figure 1. (a) Schematic representation of the computational domain for a single Taylor bubble drifting through stagnant liquid in an inclined pipe showing the initial and boundary conditions, and (b) 3D block-structured O-Grid hexahedral mesh used.

Figure 2. Streamlines and vorticity field $\left(s^{-1}\right)$ in the $X Z$ plane at the three different sections, namely: A-A, B-B, and $\mathrm{C}-\mathrm{C}$, corresponding to inclination angles of $15^{\circ}$ to $70^{\circ}$, Eo $=66$ and $\operatorname{Revd} 1 \cong 15$.

Figure 3. Sketch of the evolution of the Froude number Frvdover time for inclination angles of $15^{\circ}$ to $70^{\circ}$, Eo $=66$ and $\operatorname{Revd} 1 \cong 15$.

Figure 4. Streamlines in the $X Y$ plane for inclination angle range: $0^{\circ} \leq \theta \leq 70^{\circ}$; (a) $\operatorname{Revd} 1 \cong 15$, (b) $\operatorname{Revd} 2 \cong 95$, and (c) Terminal Froude number for the two cases, with Eo $=66$.

Figure 5. Bubble shape profile for inclination angle range: $0^{\circ} \leq \theta \leq 70^{\circ}$; (a) $\operatorname{Revd} 1 \cong 15$, (b) $\operatorname{Revd} 2 \cong$ 95 , with Eo $=66$.

Figure 6. Streamlines in the $X Y$ plane for inclination angle range: $0^{\circ} \leq \theta \leq 70^{\circ}$; (a) Eo1 $=20$, (b) Eo2 $=66$, and (c) Terminal Froude number for the two cases, with Revd $\cong 25$.

Figure 7. Bubble shape profile for inclination angle range: $0^{\circ} \leq \theta \leq 70^{\circ}$; (a) Eo1 $=20$, and (b) Eo2 $=$ 66 , with $\operatorname{Revd} \cong 25$.

Figure 8. Streamlines in $\mathrm{XY}$ plane for inclination angle range: $75^{\circ} \leq \theta<90^{\circ}$; (a) $\mathrm{GaR}=15$, (b) $\mathrm{GaR}=200$, with Eo $=66$.

Figure 9. Bubble shape profile for inclination angle range: $75^{\circ} \leq \theta<90^{\circ}$; (a) Eo $=66$, (b) Eo $=100$, with $\mathrm{GaR}=200$.

Figure 10. (a) Developed 3D Taylor bubble shape in the $X Y$ plan for inclination angle $85^{\circ}$; Eo $=66$ and $\mathrm{GaR}=200$, and (b) bubble shape profile using 3D-Transient model and 2D-Transient model.

Figure 11. (a) Developed 3D Taylor bubble shape in the $X Y$ plan for inclination angle $87^{\circ}$; Eo $=100$ and $\mathrm{GaR}=200$, and (b) bubble shape profile using 3D-Transient model and 2D-Transient model.

Figure 12. (a) Developed 3D Taylor bubble shape in the $X Y$ plan for inclination angle $89^{\circ}$; Eo $=200$ and $\mathrm{GaR}=200$, and (b) bubble shape profile using 3D-Transient model, 3D-steady model and 2Dsteady model.

Figure 13. (a) Developed 3D Taylor bubble shape in the $X Y$ plan for horizontal pipe $90^{\circ}$; Eo $=300$ and (b) bubble shape profile using 3D-steady model and 2D-steady model.

Figure 14. Effect of Eo on the bubble shape profile for the horizontal pipe; $90^{\circ}$ using 2D- steady model; for $\mathrm{Eo}=300, \mathrm{Eo}=500, \mathrm{Eo}=700$, and $\mathrm{Eo}=1000$. 
Figure 15. (a) Three dimensional Taylor bubble shape in the $X Y$ plane for all inclination angles, and (b) terminal Froude number against the experimental result of Shosho and Ryan (2001) and other correlations given in Table 2 for case 1 in Table 3.

Figure 16. Terminal Froude number against the experimental result of Shosho and Ryan (2001) and other correlations given in Table 2 for case 2 in Table 3. 
Table 1- Simulation cases used to explore the effect of both reduced Galilei number $\left(G a_{R}\right)$ and Eötvös number $(E O)$ on dynamics of Taylor bubble for inclination angle range of $70^{\circ}<\theta<90^{\circ}$, with their corresponding results.

\begin{tabular}{|c|c|c|c|c|c|}
\hline $\begin{array}{c}\text { Case } \\
\text { number }\end{array}$ & $\begin{array}{c}\text { Inclination } \\
\text { angle (degrees) }\end{array}$ & $\begin{array}{l}\text { Reduced Galilei } \\
\text { number }\left(\boldsymbol{G} \boldsymbol{a}_{R}\right)\end{array}$ & $\begin{array}{c}\text { Eötvös } \\
\text { number }(E o)\end{array}$ & $\begin{array}{c}\text { Froude } \\
\text { number } \\
\left(\boldsymbol{F} \boldsymbol{r}_{v_{d}}\right)\end{array}$ & $\left(\frac{L_{T B}}{D}\right)_{p}$ \\
\hline \multirow{2}{*}{1} & 75 & \multirow{2}{*}{15} & \multirow{4}{*}{66} & 0.0491 & 3.893 \\
\hline & 85 & & & 0.0262 & 5.053 \\
\hline \multirow{2}{*}{2} & 75 & \multirow{2}{*}{200} & & 0.1602 & 3.680 \\
\hline & 85 & & & 0.0916 & 5.032 \\
\hline \multirow{2}{*}{3} & 75 & \multirow{4}{*}{200} & \multirow{2}{*}{66} & 0.1602 & 3.680 \\
\hline & 85 & & & 0.0916 & 5.032 \\
\hline \multirow[b]{2}{*}{4} & 75 & & \multirow{2}{*}{100} & 0.1696 & 3.922 \\
\hline & 85 & & & 0.1041 & 5.505 \\
\hline
\end{tabular}

Table 2- Summary of the main correlations proposed in the literature for the rising velocity of a Taylor bubble in inclined pipes with stagnant liquid.

\begin{tabular}{|c|c|c|}
\hline $\begin{array}{c}\text { Reference/ } \\
\text { Type of study }\end{array}$ & Correlation & Assumptions and comments \\
\hline $\begin{array}{l}\text { Bendiksen (1985) } \\
\text { Experimental and } \\
\text { theoretical }\end{array}$ & $v_{d}=v_{d}^{h} \cos \theta+v_{d}^{v} \sin \theta, v_{d}^{h}=0.351 \sqrt{g D}, v_{d}^{v}=0.542 \sqrt{g D}$ & $\begin{array}{l}\text { - } \theta \text { ranges from }-90^{\circ} \text { to } 90^{\circ} . \\
\text { - } \quad E o \text { ranges from } 50-340, M \approx 2.63 \cdot 10^{-11} \\
\text { - } F r_{v_{\text {max }}} \text { at } 40^{\circ}<\theta<60^{\circ}\end{array}$ \\
\hline $\begin{array}{l}\text { Weber et al. (1986) } \\
\text { Experimental }\end{array}$ & $F r_{v_{d}}=F r_{v_{d}}{ }^{h} \cos \theta+F r_{v_{d}}{ }^{v} \sin \theta+Q, Q= \begin{cases}1.37\left(\Delta F r_{v_{d}}\right)^{\frac{2}{3}} \sin \theta(1-\sin \theta), & \text { if } \Delta F r_{v_{d}}>0 \\
0, & \text { if } \Delta F r_{v_{d}} \leq 0\end{cases}$ & $\begin{array}{l}\text { - } \theta \text { ranges from } 0 \text { to } 90^{\circ} \text { from horizontal position. } \\
\text { - } E o \text { ranges from } 4.9-490, M=2.2 \cdot 10^{-11}-1.5 \cdot 10^{4} \\
\text { - } F r_{v_{\max }} \text { at } 30^{\circ}<\theta<60^{\circ} \\
\text { - } \quad \text { igh-viscosity Newtonian liquids. }\end{array}$ \\
\hline $\begin{array}{l}\text { Hasan and Kabir } \\
\text { (1988) } \\
\text { Experimental and } \\
\text { theoretical }\end{array}$ & $v_{d}=v_{d}^{v} \sqrt{\sin \theta}(1+\cos \theta)^{1.2}, v_{d}^{v}=0.35 \sqrt{g D}, v_{d}^{h}=0$ & $\begin{array}{l}\text { - } \theta \text { ranges from } 30 \text { to } 90^{\circ} \text { from horizontal position. } \\
\text { - } A \text { Assume } v_{d}{ }^{h}=0 \text {. } \\
\text { - Applicable for systems with large } N_{f} \text { and } E o \text { values } \\
\text { - } E o=2200, M=2.63 \cdot 10^{-11}\end{array}$ \\
\hline $\begin{array}{l}\text { Petalas and Aziz } \\
(2000) \\
\text { Theoretical }\end{array}$ & $\begin{array}{l}\text { - } \quad v_{d}{ }^{h}=\left(0.54-\frac{1.76}{E 0_{0.56}}\right) \sqrt{\frac{\sqrt{g\left(\rho_{L}-\rho_{G}\right)}}{\rho_{L}}}, v_{d}{ }^{v}=0.345\left(1-e^{-E o-\exp (3.278-1.424 \ln E o)}\right) \sqrt{\frac{g D\left(\rho_{L}-\rho_{G}\right)}{\rho_{L}}} \\
\text { - } \quad v_{d}=f_{m}\left(v_{d}{ }^{h} \cos \theta+v_{d}{ }^{v} \sin \theta\right), f_{m}=\min \left(0.316 \sqrt{\frac{v_{d_{\infty} D \rho_{L}}}{2 \mu_{L}}}, 1\right)(\text { Zukoski }(1966))\end{array}$ & $\begin{array}{l}\text { - } \theta \text { ranges from }-90^{\circ} \text { to } 90^{\circ} . \\
\text { - } F r_{v_{\text {max }}} \text { at } 40^{\circ} \\
\text { - } \quad \text { Applicable for high Reynolds numbers }\end{array}$ \\
\hline $\begin{array}{l}\text { Gokcal et al. } \\
(2009) \\
\text { Experimental }\end{array}$ & $\begin{array}{ll}\text { - } & h / D=0.1038 \ln \mu_{L}+0.9684, S / D=\sqrt{1-(2 h / D-1)^{2}} \\
\text { - } & \gamma_{1}=\left\{\begin{array}{l}\pi-\sin ^{-1}(S / D) \text { if } h / D<0.5 \\
\sin ^{-1}(S / D) \quad \text { if } h / D \geq 0.5^{\prime}\end{array}\right. \\
\text { - } & \gamma=\min \left(\gamma_{1}, 1.444784\right), \zeta=(\gamma-0.5 \sin 2 \gamma) / \pi \\
\text { - } & \Delta=\frac{1+\zeta}{\zeta}\left(\left(\frac{D}{2}(1-\cos \gamma)\right)-\left(\frac{\left(\frac{D}{2}(1-(1-\zeta) \cos \gamma)+\frac{2}{3 \pi} \sin \gamma\right.}{1-\zeta^{2}}\right)\right) \times 2.2 \\
\text { - } & v_{2}=\sqrt{g D\left((1-(1-\zeta) \cos \gamma)-\frac{2}{3 \pi} \sin ^{3} \gamma\right)-2 \Delta g(1-\zeta)} \\
\text { - } & v_{d}^{h}=v_{1}=(1+\zeta) v_{2}, v_{d}^{v}=\frac{-8}{3} \frac{\mu_{L}}{\rho_{L} D}+\sqrt{\frac{2}{9} g D+\frac{64}{9}\left(\frac{\mu_{L}}{\rho_{L} D}\right)^{2}} \\
\text { - } & v_{d}=v_{d}^{h}(\cos \theta)^{1.5}+v_{d}^{v}(\sin \theta)^{0.7}, \zeta=(\gamma-0.5 \sin 2 \gamma) / \pi \\
\text { - } & \Delta=\frac{1+\zeta}{\zeta}\left(\left(\frac{D}{2}(1-\cos \gamma)\right)-\left(\frac{\left(\frac{D}{2}(1-(1-\zeta) \cos \gamma)+\frac{2}{3 \pi} \sin \gamma\right.}{1-\zeta^{2}}\right)\right) \times 2.2 \\
\text { - } & v_{2}=\sqrt{g D\left((1-(1-\zeta) \cos \gamma)-\frac{2}{3 \pi} \sin ^{3} \gamma\right)-2 \Delta g(1-\zeta)} \\
\text { - } & v_{d}^{h}=v_{1}=(1+\zeta) v_{2}, v_{d}{ }^{v}=\frac{-8}{3} \frac{\mu_{L}}{\rho_{L} D}+\sqrt{\frac{2}{9} g D+\frac{64}{9}\left(\frac{\mu_{L}}{\rho_{L} D}\right)^{2}} \\
\text { - } & v_{d}=v_{d}^{h}(\cos \theta)^{1.5}+v_{d}^{v}(\sin \theta)^{0.7} \\
\end{array}$ & $\begin{array}{l}\text { - } \theta \text { ranges from } 5 \text { to } 90^{\circ} \text { measured from the } \\
\text { horizontal position. } \\
\text { - } E o=776, M=4.52 \cdot 10^{-10}-1.63 \cdot 10^{3} \\
\text { - } F r_{v_{\text {max }} \text { at }} 40^{\circ} \text {. } \\
\text { - } \text { Correlation applicable for high viscosity oil. }\end{array}$ \\
\hline $\begin{array}{l}\text { Jeyachandra et al. } \\
\text { (2012) } \\
\text { Experimental }\end{array}$ & $\begin{array}{l}F r_{v_{d}}=F r_{v_{d}}{ }^{h} \cos \theta+F r_{v_{d}}{ }^{v} \sin \theta, F r_{v_{d}}{ }^{h}=0.53 \exp \left(-13.7 N_{f}^{-0.46} \mathrm{Eo}^{-0.1}\right) \\
F r_{v_{d}}{ }^{v}=\frac{-8}{3} N_{f}^{-1}+\sqrt{\frac{2}{9} \frac{\rho_{L}}{\left(\rho_{L}-\rho_{G}\right)}+\frac{64}{9} N_{f}^{-2}} .\end{array}$ & $\begin{array}{l}\text { - } \theta \text { ranges from } 0 \text { to } 90^{\circ} \text { measured from the } \\
\text { horizontal position } \\
\text { - } E o=776-6985, M=0.254-49.1 \\
\text { - } F r_{v_{\text {max }}} \text { at } 30^{\circ}<\theta<50^{\circ}\end{array}$ \\
\hline $\begin{array}{l}\text { Moreiras et al. } \\
(2014) \\
\text { Experimental }\end{array}$ & $\begin{array}{ll}\text { - } F r_{v_{d}}=F r_{v_{d}}{ }^{h} \cos ^{a} \theta+F r_{v_{d}}{ }^{v} \sin ^{b} \theta+Q, F v_{v_{d}}{ }^{b}=\frac{-8}{3} N_{f}^{-1}+\sqrt{\frac{2}{9} \frac{\rho_{L}}{\left(\rho_{L}-\rho_{G}\right)}+\frac{64}{9} N_{f}^{-2}}-\left(\frac{\sqrt{2}}{3}-0.35\right) \sqrt{\frac{\rho_{L}}{\left(\rho_{L}-\rho_{G}\right)^{\prime}}} \\
\text { - } F r_{v_{d}}{ }^{h}=0.54-\frac{N_{f}^{-1}}{1.886+0.01443 N_{f}^{-1}}, Q= \begin{cases}c\left(\Delta F r_{v_{d}}\right)^{d} \sin \theta(1-\sin \theta), & \text { if } \Delta F r_{v_{d}} \geq 0 \\
0, & \text { if } \Delta F r_{v_{d}}<0\end{cases} \\
\text { - } \quad a=1.2391, b=1.2315, c=2.1589, d=0.70412 & \end{array}$ & $\begin{array}{l}\text { - } \theta \text { ranges from } 0 \text { to } 90^{\circ} \text { measured from the } \\
\text { horizontal position } \\
\text { - } E o=804, M=1.25 \cdot 10^{-3}-10.412 \\
\text { - } F r_{v_{\text {max }}} \text { at } 30^{\circ}<\theta<50^{\circ}\end{array}$ \\
\hline $\begin{array}{l}\text { Lizarraga-García } \\
\text { (2016) } \\
\text { Experimental and } \\
\text { Numerical work }\end{array}$ & 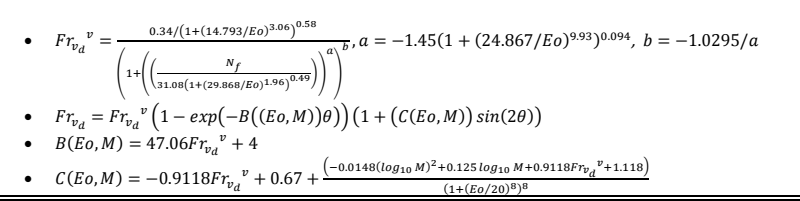 & $\begin{array}{l}\text { - } \theta \text { ranges from } 5 \text { to } 90^{\circ} \text { measured from the } \\
\text { horizontal position. } \\
\text { - } \text { The horizontal pipe case is not included. } \\
\text { - } E o=10: 700, M=1 \cdot 10^{-6}-5 \cdot 10^{3} \\
\text { - } F r_{v_{\text {max }}} \text { at } 45^{\circ} \text {. }\end{array}$ \\
\hline
\end{tabular}


Table 3- Test matrix for simulations of drift of Taylor bubble through stagnant liquid in inclined pipes based on the experimental work of Shosho and Ryan (2001).

(a)

(b)

Figure 1. (a) Schematic representation of the computational domain for a single Taylor bubble drifting through stagnant liquid in an inclined pipe showing the initial and boundary conditions, and (b) 3D blockstructured O-Grid hexahedral mesh used. 


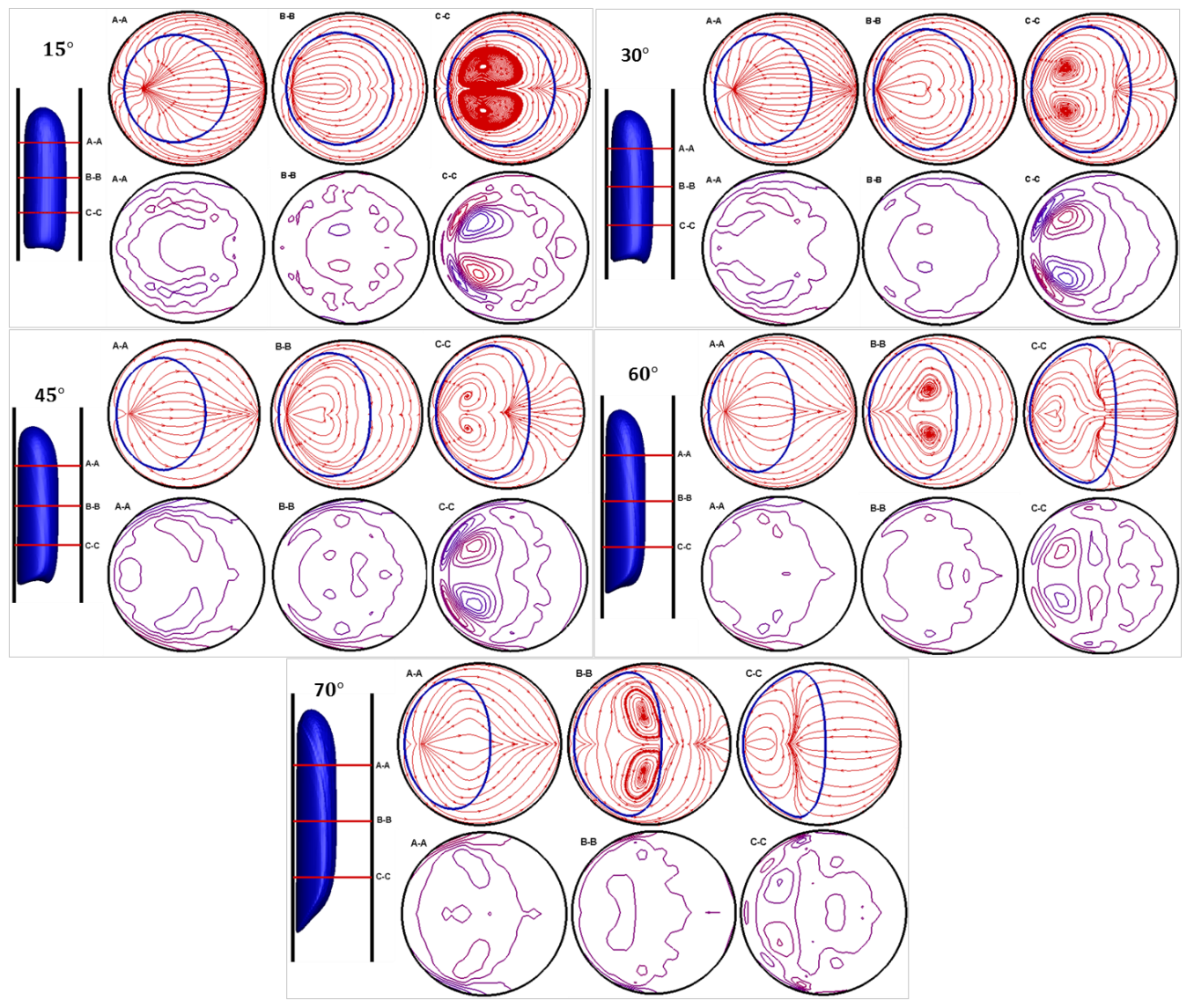

Figure 2. Streamlines and vorticity field $\left(\mathrm{s}^{-1}\right)$ in the $\mathrm{XZ}$ plane at the three different sections, namely: AA, B-B, and C-C, corresponding to inclination angles of $15^{\circ}$ to $70^{\circ}, E o=66$ and $R e_{v_{\mathrm{d}_{1}}} \cong 15$.

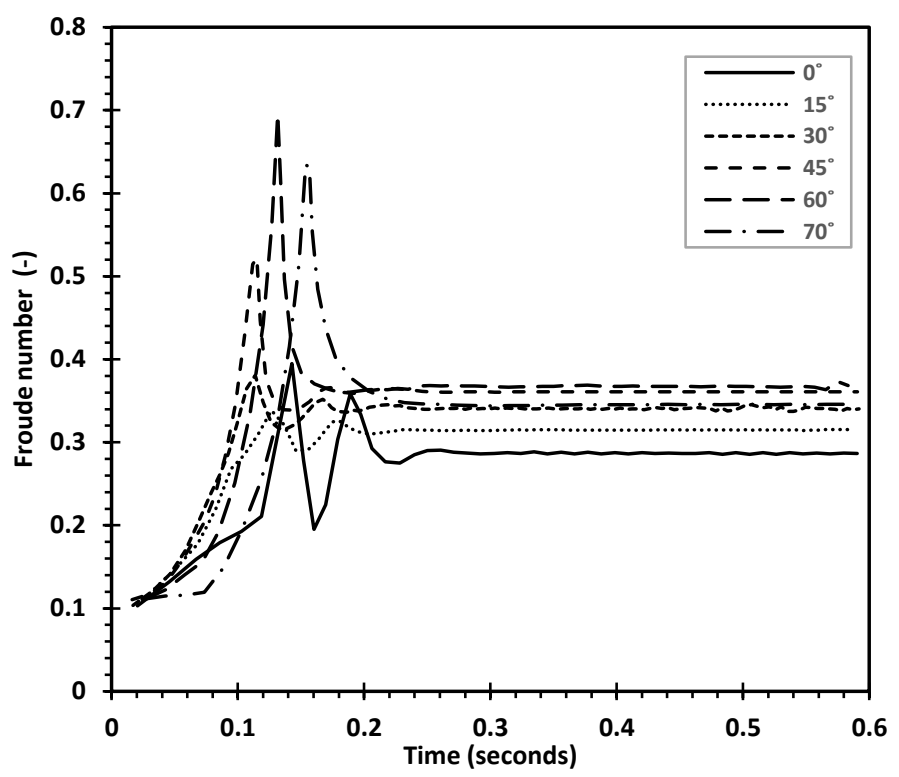

Figure 3. Sketch of the evolution of the Froude number $F r_{v_{d}}$ over time for inclination angles of $15^{\circ}$ to $70^{\circ}, E o=66$ and $R e_{v_{\mathrm{d}_{1}}} \cong 15$. 


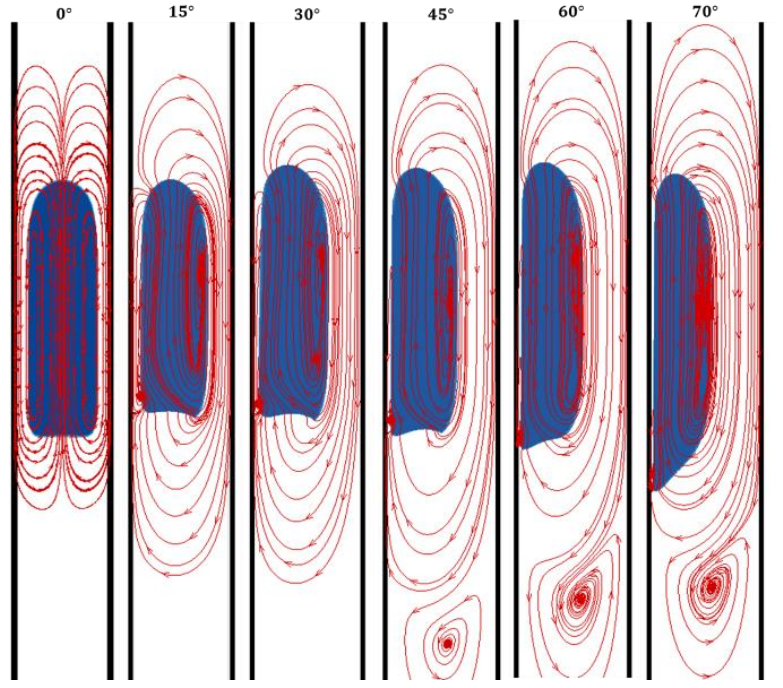

(a)

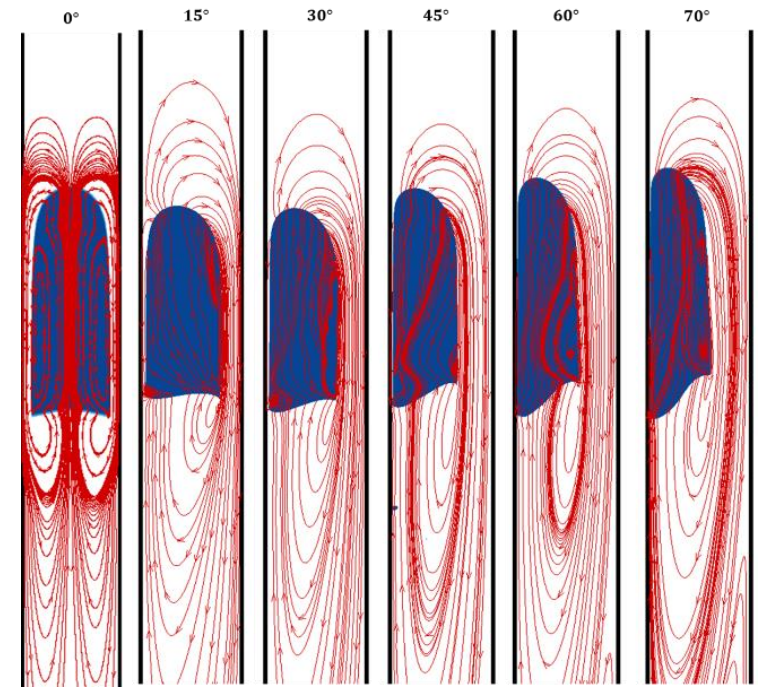

(b)

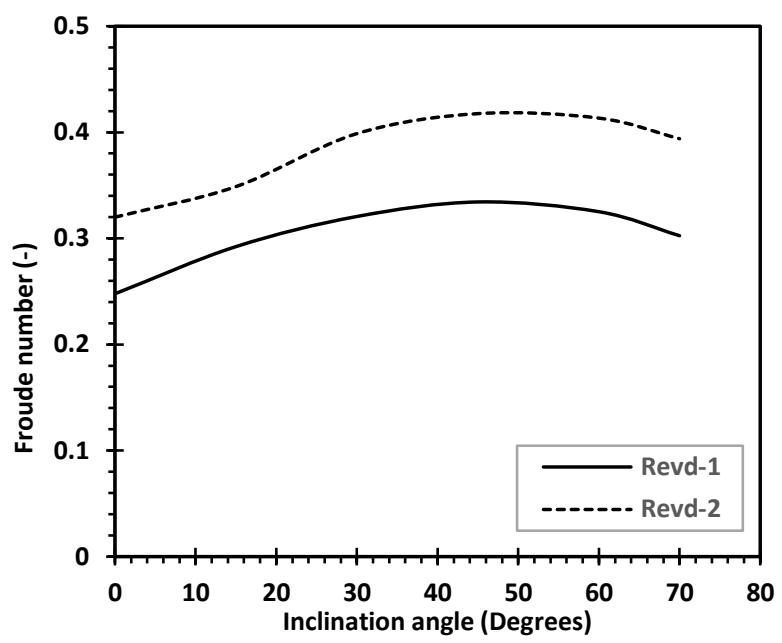

(c)

Figure 4. Streamlines in the XY plane for inclination angle range: $0^{\circ} \leq \theta \leq 70^{\circ}$; (a) $R e_{v_{\mathrm{d}_{1}}} \cong 15$, (b) $R e_{v_{\mathrm{d} 2}} \cong 95$, and (c) Terminal Froude number for the two cases, with $E o=66$. 


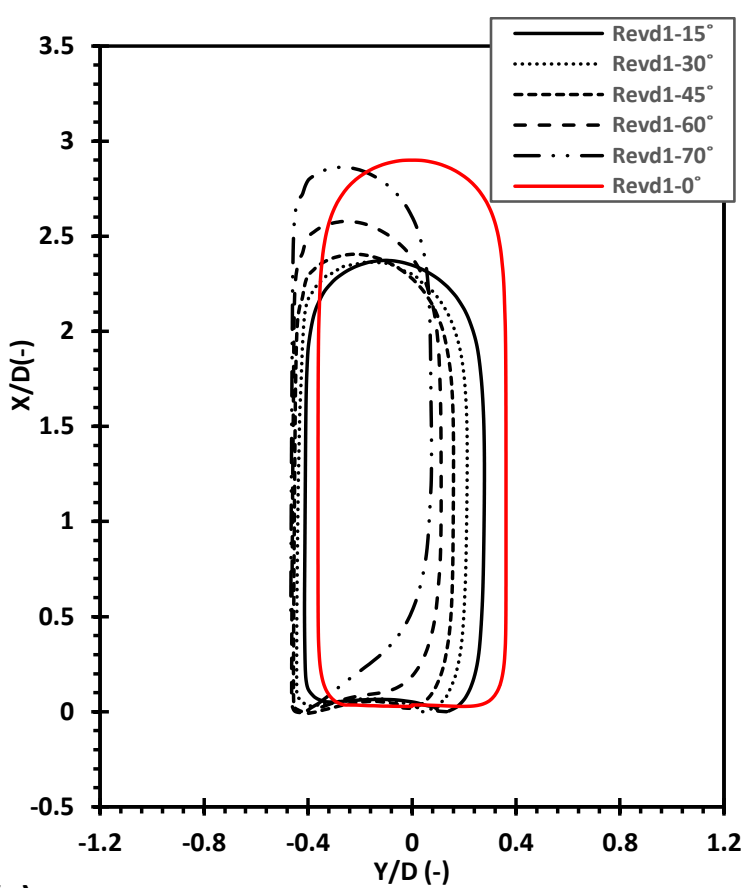

(a)

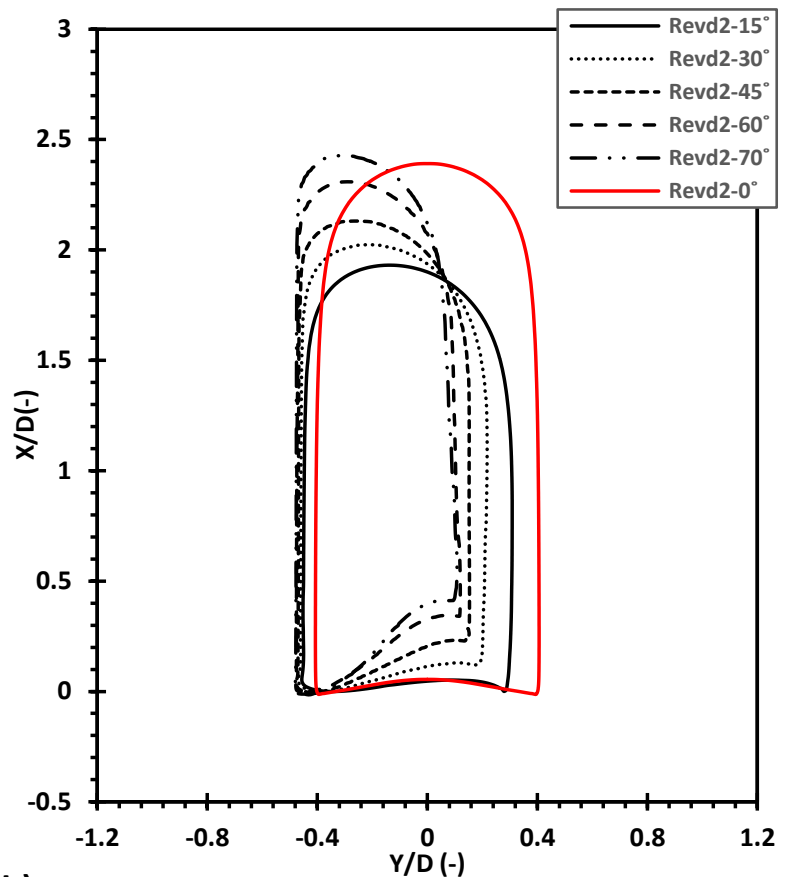

(b)

Figure 5. Bubble shape profile for inclination angle range: $0^{\circ} \leq \theta \leq 70^{\circ}$; (a) $R e_{v_{d_{1}}} \cong 15$, (b) $R e_{v_{d_{2}}} \cong$ 95, with $E o=66$. 


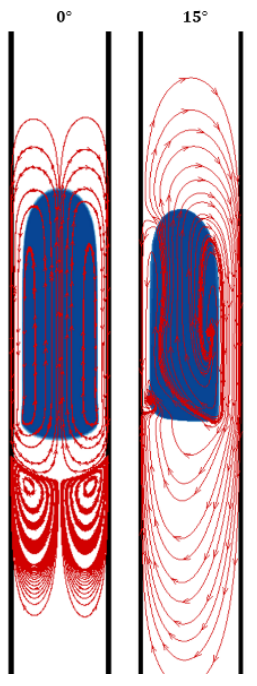

(a)
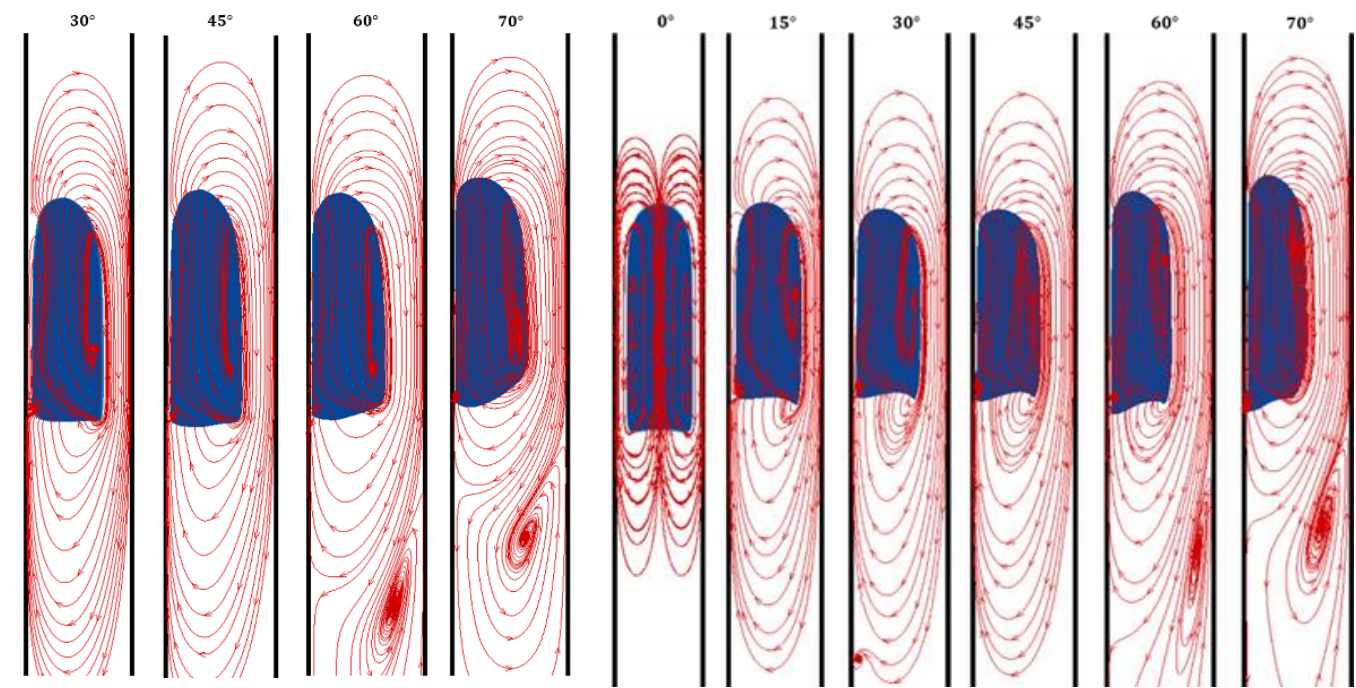

(b)

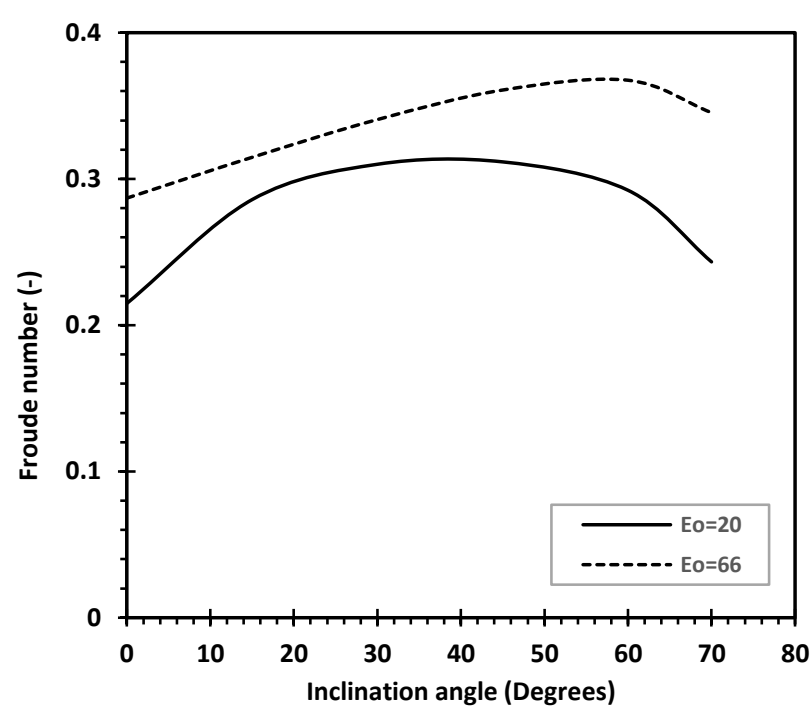

(c)

Figure 6. Streamlines in the XY plane for inclination angle range: $0^{\circ} \leq \theta \leq 70^{\circ}$; (a) $E o_{1}=20$, (b) $E o_{2}=$ 66, and (c) Terminal Froude number for the two cases, with $R e_{v_{\mathrm{d}}} \cong 25$. 


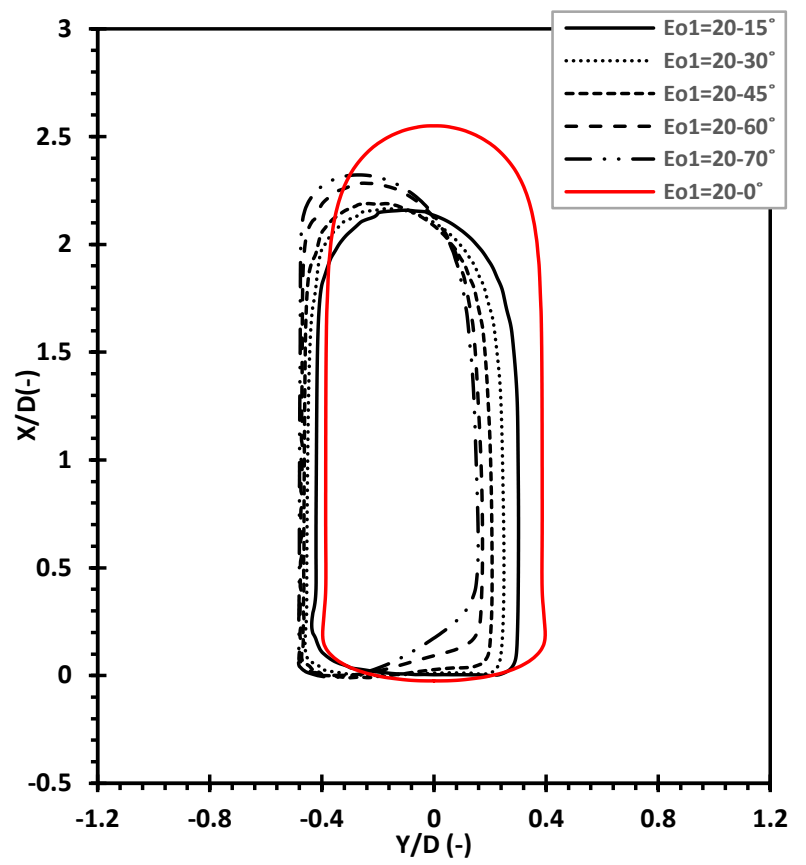

(a)

Figure 7. Bubble shape profile for inclination angle range: $0^{\circ} \leq \theta \leq 70^{\circ}$; (a) $E o_{1}=20$, and (b) $E o_{2}=$ 66, with $R e_{v_{\mathrm{d}}} \cong 25$.

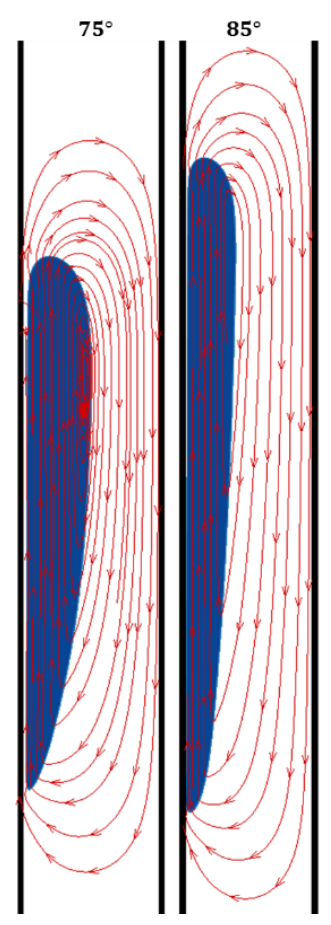

(b)

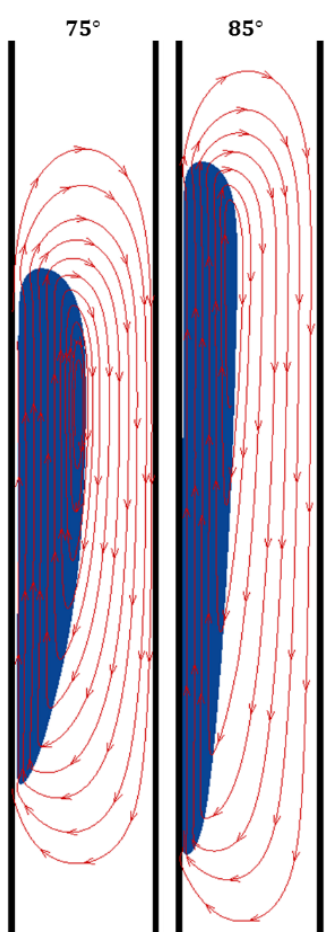

Figure 8. Streamlines in XY plane for inclination angle range: $75^{\circ} \leq \theta<90^{\circ}$; (a) $G a_{R}=15$, (b) $G a_{R}=$ 200, with $E o=66$.

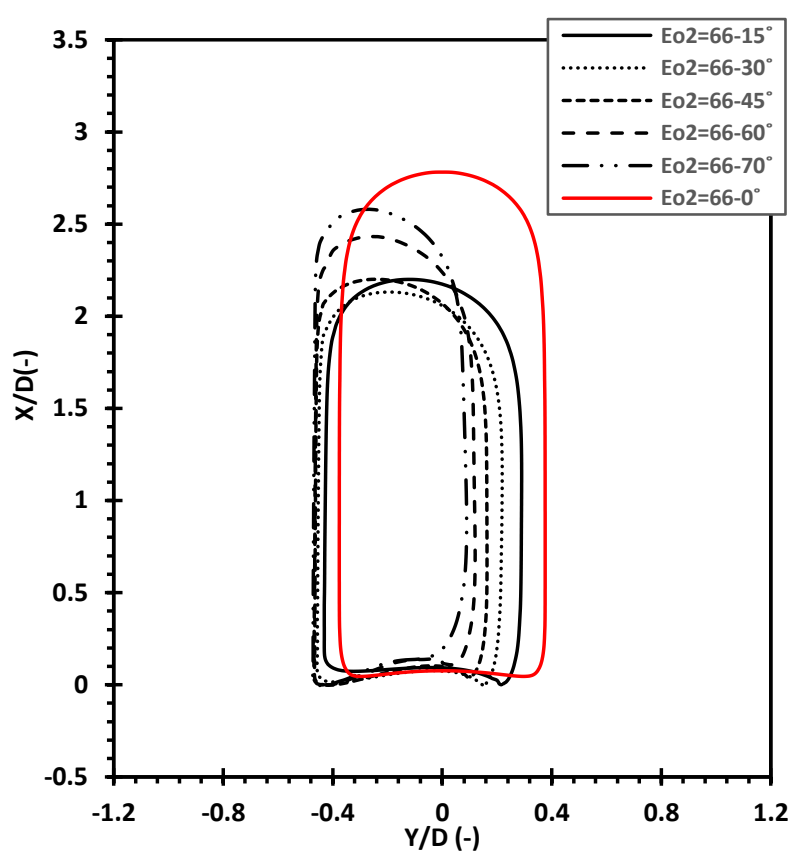

(b) 


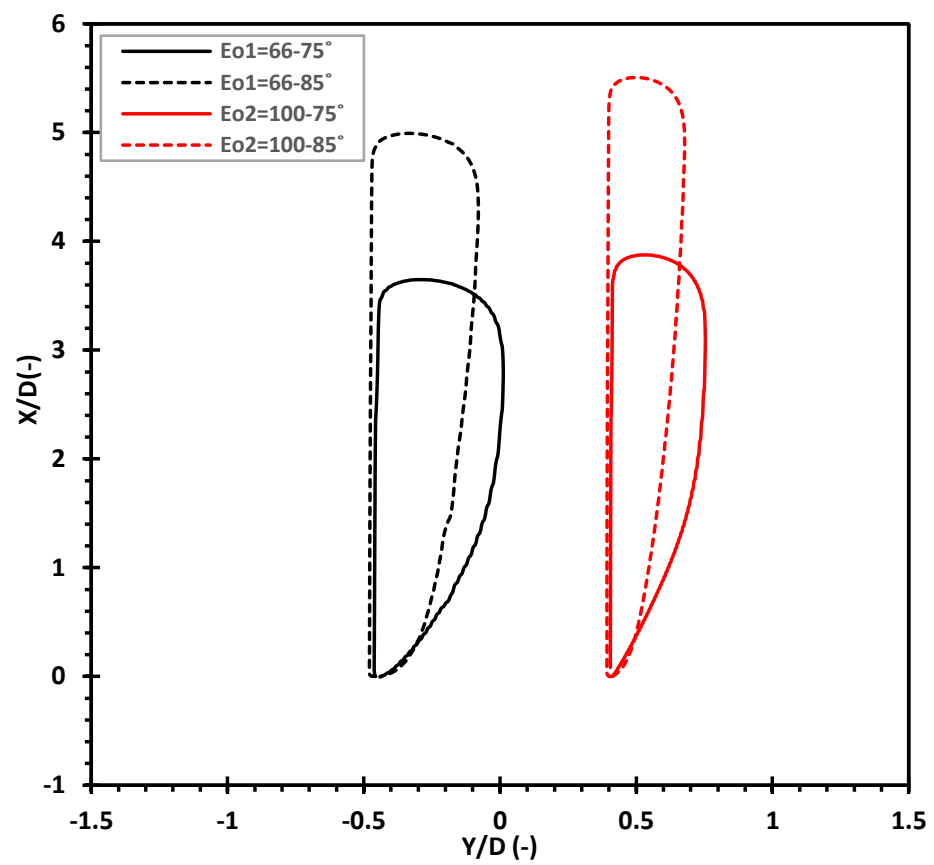

Figure 9. Bubble shape profile for inclination angle range: $75^{\circ} \leq \theta<90^{\circ}$; (a) $E o=66$, (b) $E o=100$, with $G a_{R}=200$.

(a)

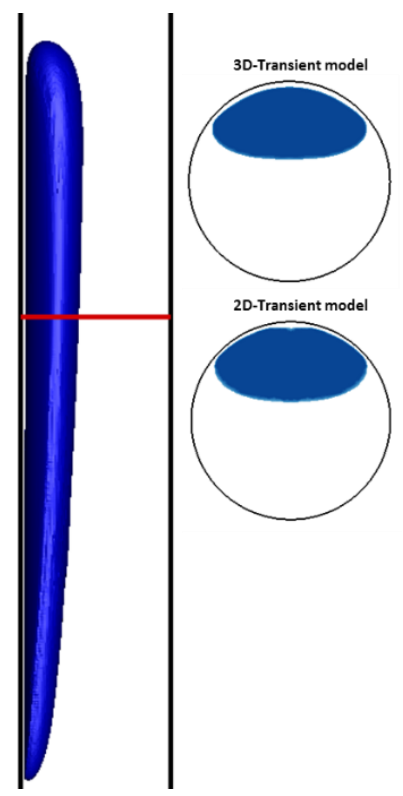

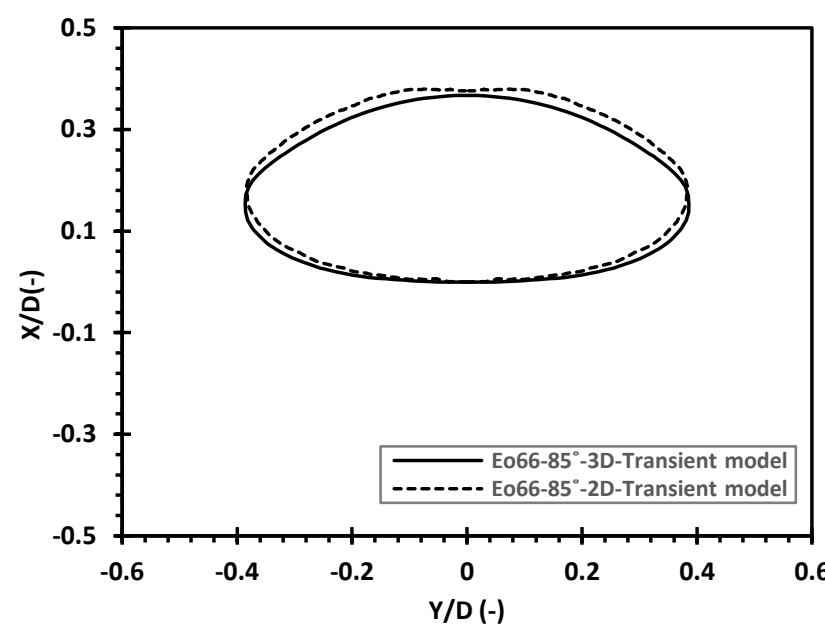

(b)

Figure 10. (a) Developed 3D Taylor bubble shape in the XY plan for inclination angle $85^{\circ}$; Eo $=66$ and $G a_{R}=200$, and (b) bubble shape profile using 3D-Transient model and 2D-Transient model. 


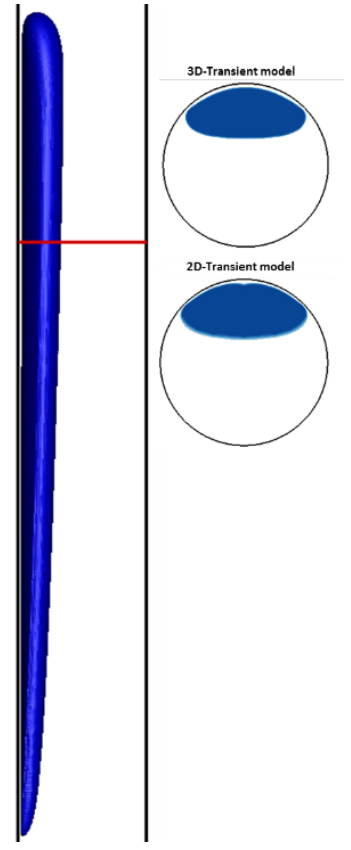

(a)

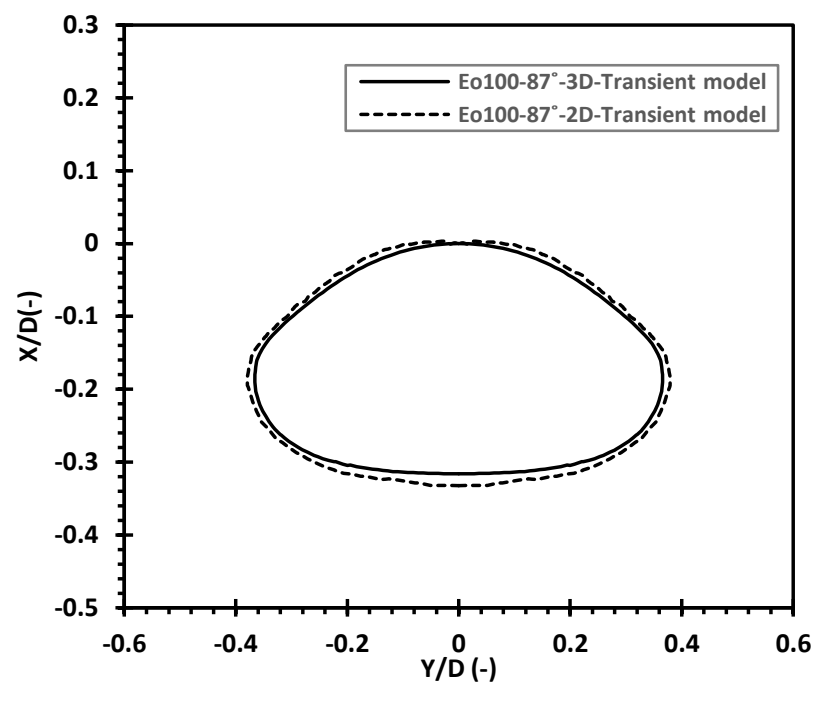

(b)

Figure 11. (a) Developed 3D Taylor bubble shape in the XY plan for inclination angle $87^{\circ} ; E o=100$ and $G a_{R}=200$, and (b) bubble shape profile using 3D-Transient model and 2D-Transient model.

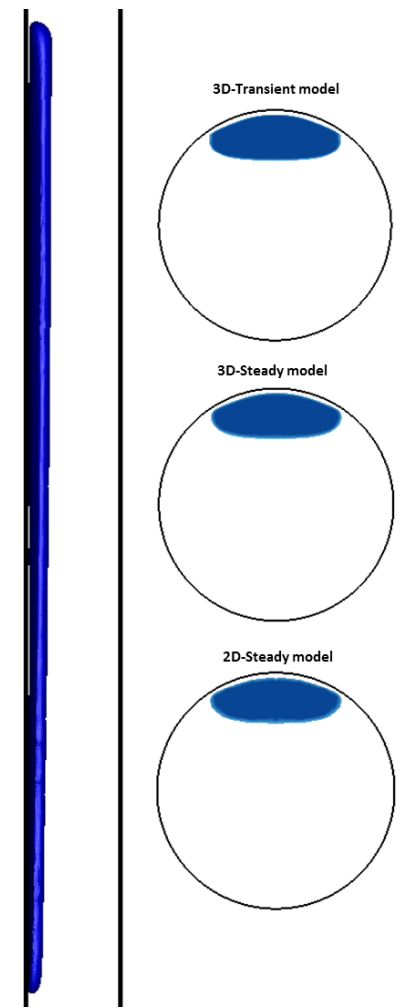

(a)

Figure 12. (a) Developed 3D Taylor bubble shape in the XY plan for inclination angle $89^{\circ} ; E o=200$ and $G a_{R}=200$, and (b) bubble shape profile using 3D-Transient model, 3D-steady model and 2D-steady model.

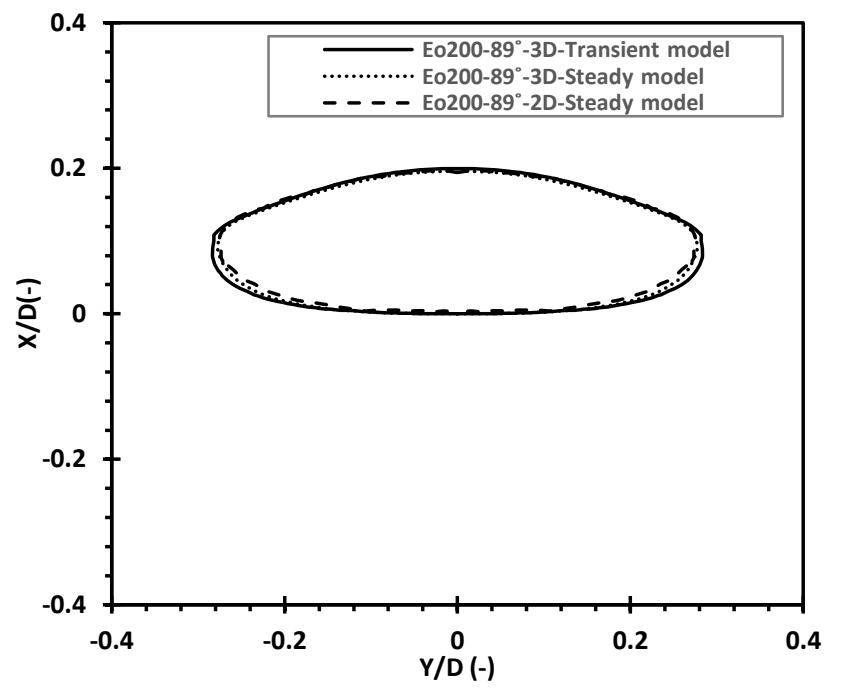

(b) 
(a)
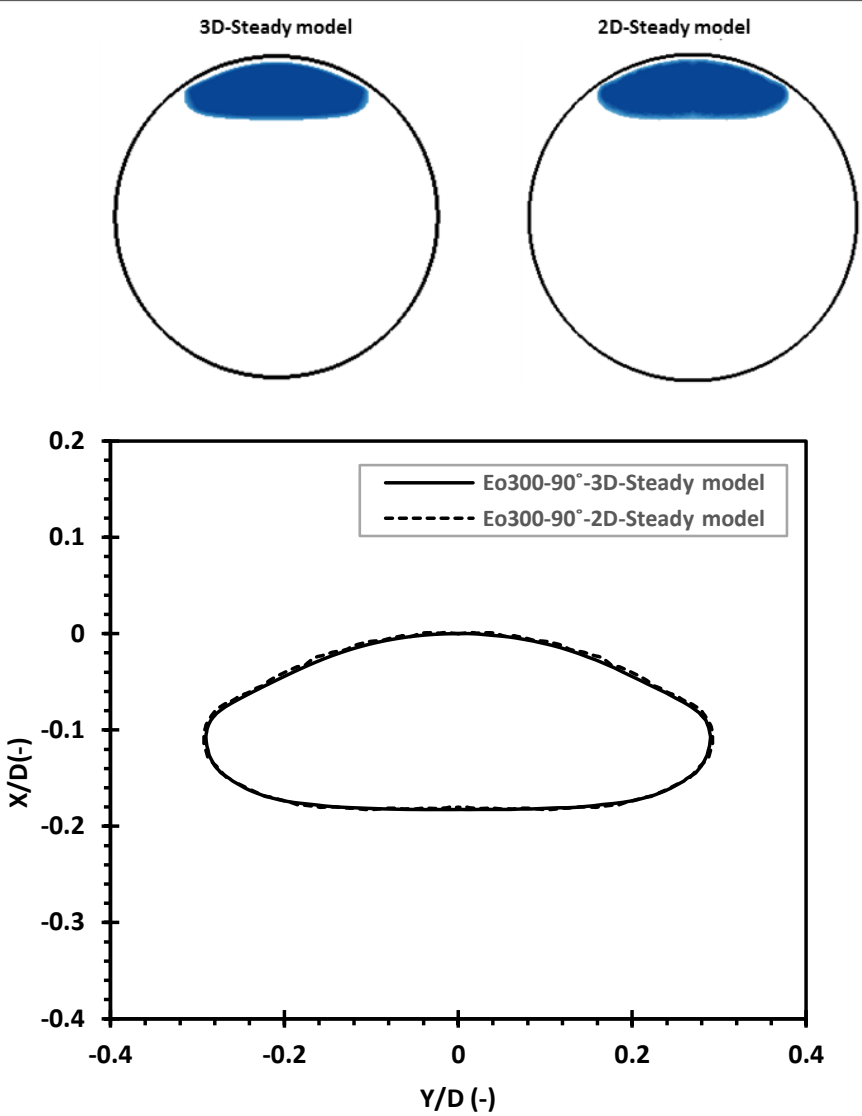

Figure 13. (a) Developed 3D Taylor bubble shape in the XY plan for horizontal pipe $\left(90^{\circ}\right) ; E o=300$ and (b) bubble shape profile using 3D-steady model and 2D-steady model.

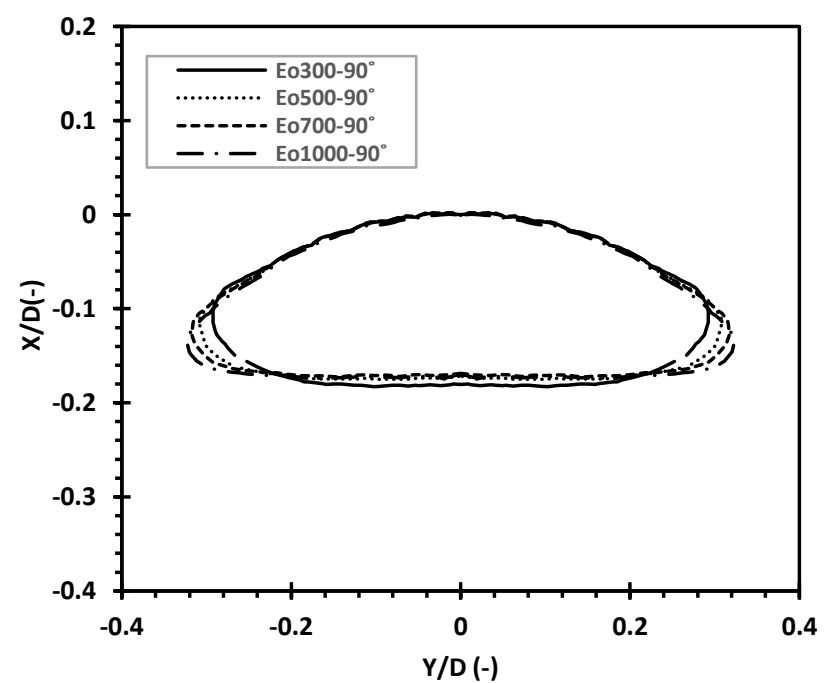

Figure 14. Effect of $E o$ on the bubble shape profile for the horizontal pipe; $90^{\circ}$ using 2D- steady model; for $E o=300, E o=500, E o=700$, and $E o=1000$. 
(a)

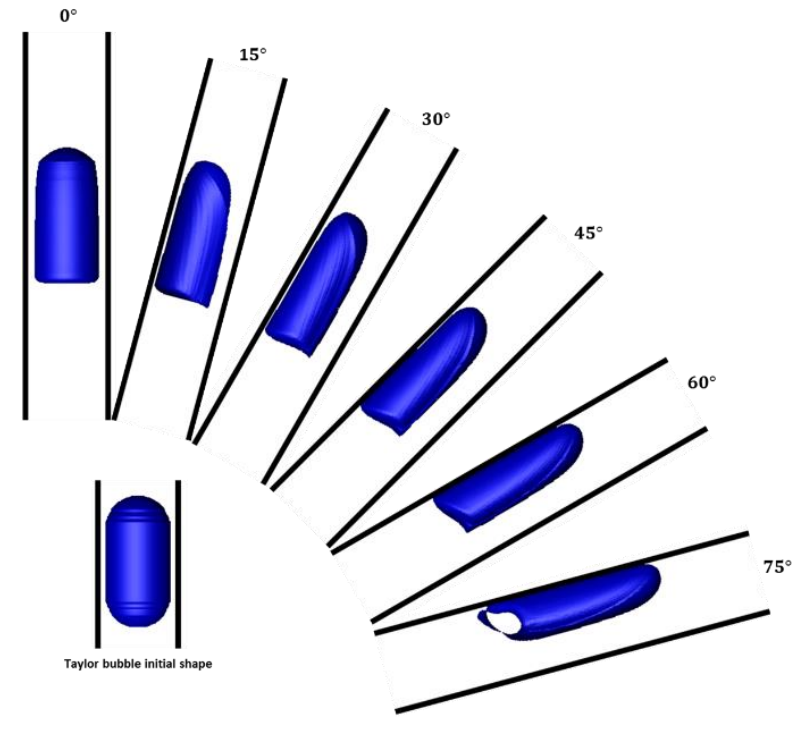

(b)

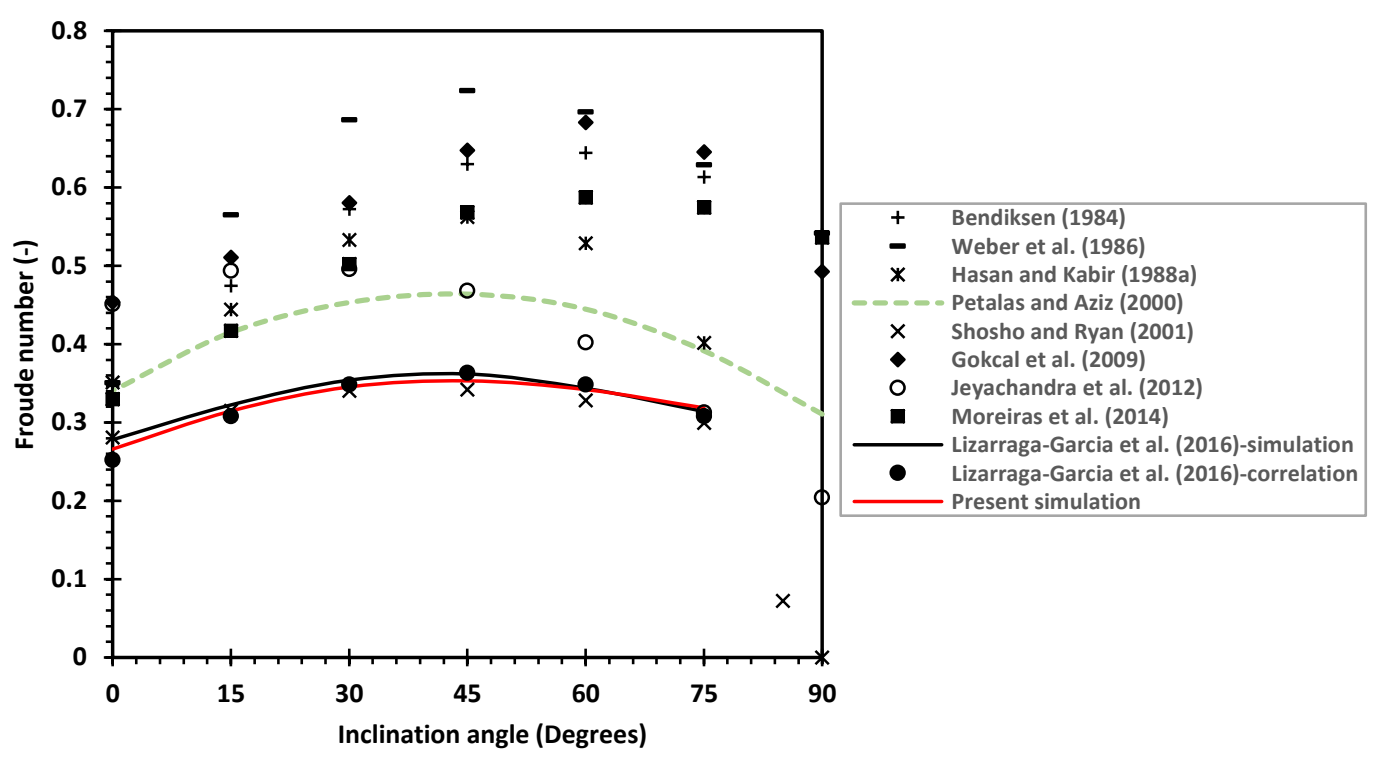

Figure 15. (a) Three dimensional Taylor bubble shape in the XY plane for all inclination angles, and (b) terminal Froude number against the experimental result of Shosho and Ryan (2001) and other correlations given in Table 2 for case 1 in Table 3. 


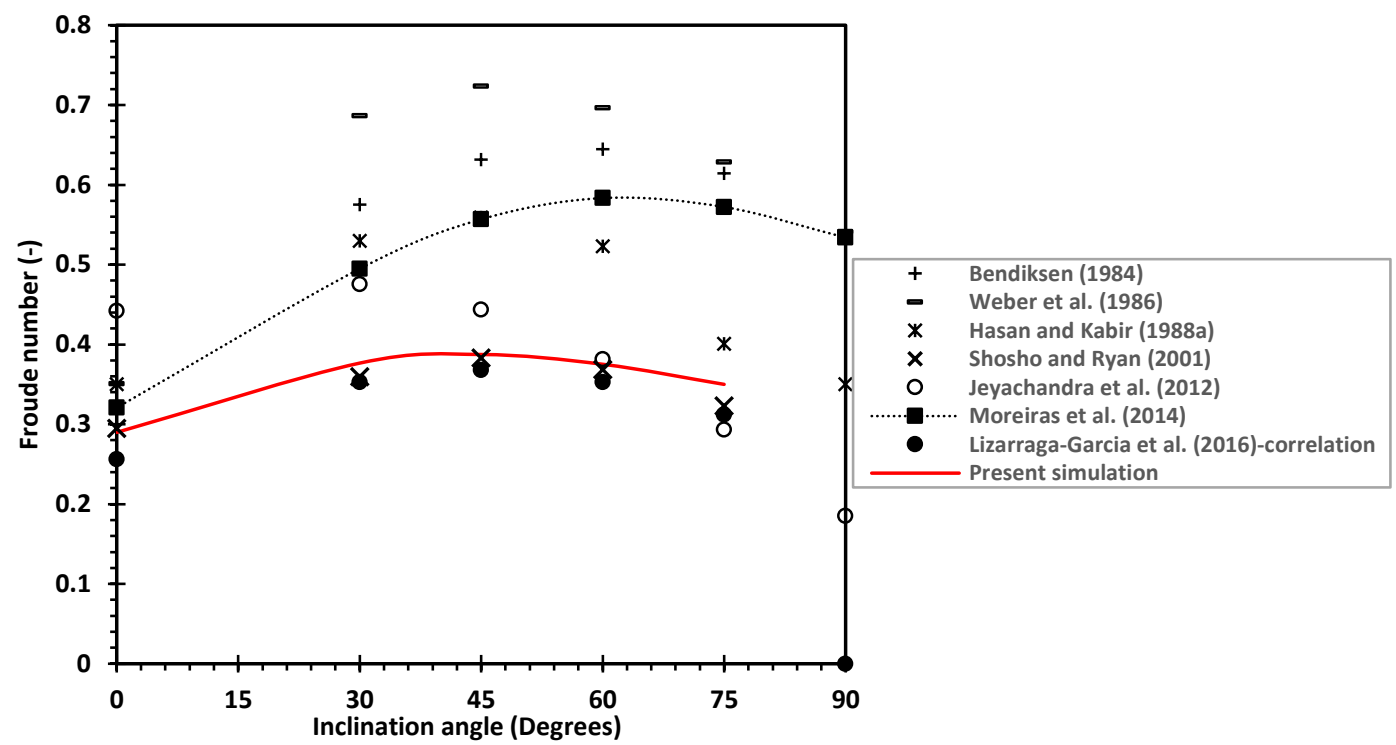

Figure 16. Terminal Froude number against the experimental result of Shosho and Ryan (2001) and other correlations given in Table 2 for case 2 in Table 3.

\section{Appendix 1- Order of magnitude analysis for the equations of motion}

The governing equations of the problem are the continuity and momentum equations expressed in Cartesian coordinates. With liquid phase identified as $L$, the Navier-stokes equations in cartesian coordinates are:

$\rho_{L}\left(\frac{\partial u}{\partial t}+u \frac{\partial u}{\partial x}+v \frac{\partial u}{\partial y}+w \frac{\partial u}{\partial z}\right)=\rho_{L} g_{x}-\frac{\partial p}{\partial x}+\mu_{L}\left[\frac{\partial^{2} u}{\partial x^{2}}+\frac{\partial^{2} u}{\partial y^{2}}+\frac{\partial^{2} u}{\partial z^{2}}\right]$

$\rho_{L}\left(\frac{\partial v}{\partial t}+u \frac{\partial v}{\partial x}+v \frac{\partial v}{\partial y}+w \frac{\partial v}{\partial z}\right)=\rho_{L} g_{y}-\frac{\partial p}{\partial y}+\mu_{L}\left[\frac{\partial^{2} v}{\partial x^{2}}+\frac{\partial^{2} v}{\partial y^{2}}+\frac{\partial^{2} v}{\partial z^{2}}\right]$

$\rho_{L}\left(\frac{\partial w}{\partial t}+u \frac{\partial w}{\partial x}+v \frac{\partial w}{\partial y}+w \frac{\partial w}{\partial z}\right)=\rho_{L} g_{z}-\frac{\partial p}{\partial z}+\mu_{L}\left[\frac{\partial^{2} w}{\partial x^{2}}+\frac{\partial^{2} w}{\partial y^{2}}+\frac{\partial^{2} w}{\partial z^{2}}\right]$

And the continuity equation is:

$\frac{\partial u}{\partial x}+\frac{\partial v}{\partial y}+\frac{\partial w}{\partial z}=0$

where $L$ stands for the liquid phase.

Introducing the following dimensionless variables, with characteristic drift velocity $\left(v_{d}\right)$ :

$u^{*}=\frac{u}{v_{d}}, v^{*}=\frac{v}{v_{d}}, w^{*}=\frac{w}{v_{d}}, p^{*}=\frac{p}{\frac{1}{2} \rho_{L} v_{d}^{2}}, x^{*}=\frac{x}{(D / 2)}, y^{*}=\frac{y}{(D / 2)}, z^{*}=\frac{z}{L_{T B}}$, 
$t^{*}=t\left(\frac{v_{d}}{L_{T B}}\right), g_{x}{ }^{*}=\frac{g_{x}}{g}, g_{y}{ }^{*}=\frac{g_{y}}{g}, g_{z}{ }^{*}=\frac{g_{z}}{g}, \sigma_{x}{ }^{*}=\frac{\sigma_{x}}{\sigma}, \sigma_{y}^{*}=\frac{\sigma_{y}}{\sigma}, \sigma_{z}^{*}=\frac{\sigma_{z}}{\sigma}$,

$K^{*}=\frac{K}{\left(1 / D^{2}\right)}$

The Navier-stokes and continuity equations in dimensionless form can be summarised as follows:

$$
\begin{gathered}
\left(\frac{D}{L_{T B}}\right) \frac{\partial u^{*}}{\partial t^{*}}+2 u^{*} \frac{\partial u^{*}}{\partial x^{*}}+2 v^{*} \frac{\partial u^{*}}{\partial y^{*}}+\left(\frac{D}{L_{T B}}\right) w^{*} \frac{\partial u^{*}}{\partial z^{*}} \\
=\frac{g_{x}^{*}}{F r_{v_{d}}^{2}}-\frac{\partial p^{*}}{\partial x^{*}}+\frac{1}{R e_{v_{d}}}\left[4 \frac{\partial^{2} u^{*}}{\partial x^{* 2}}+4 \frac{\partial^{2} u^{*}}{\partial y^{* 2}}+\left(\frac{D}{L_{T B}}\right)^{2} \frac{\partial^{2} u^{*}}{\partial z^{* 2}}\right] \\
\left(\frac{D}{L_{T B}}\right) \frac{\partial v^{*}}{\partial t^{*}}+2 u^{*} \frac{\partial v^{*}}{\partial x^{*}}+2 v^{*} \frac{\partial v^{*}}{\partial y^{*}}+\left(\frac{D}{L_{T B}}\right) w^{*} \frac{\partial v^{*}}{\partial z^{*}} \\
=\frac{g_{y}{ }^{*}}{F r_{v_{d}}^{2}}-\frac{\partial p^{*}}{\partial y^{*}}+\frac{1}{R e_{v_{d}}}\left[4 \frac{\partial^{2} v^{*}}{\partial x^{* 2}}+4 \frac{\partial^{2} v^{*}}{\partial y^{* 2}}+\left(\frac{D}{L_{T B}}\right)^{2} \frac{\partial^{2} v^{*}}{\partial z^{* 2}}\right] \\
\left(\frac{D}{L_{T B}}\right) \frac{\partial w^{*}}{\partial t^{*}}+2 u^{*} \frac{\partial w^{*}}{\partial x^{*}}+2 v^{*} \frac{\partial w^{*}}{\partial y^{*}}+\left(\frac{D}{L_{T B}}\right) w^{*} \frac{\partial w^{*}}{\partial z^{*}} \\
=\frac{g_{z}^{*}}{F r_{v_{d}}^{2}}-\frac{\partial p^{*}}{\partial z^{*}}+\frac{1}{R e_{v_{d}}}\left[4 \frac{\partial^{2} w^{*}}{\partial x^{* 2}}+4 \frac{\partial^{2} w^{*}}{\partial y^{* 2}}+\left(\frac{D}{L_{T B}}\right)^{2} \frac{\partial^{2} w^{*}}{\partial z^{* 2}}\right]
\end{gathered}
$$

$\frac{\partial u^{*}}{\partial x^{*}}+\frac{\partial v^{*}}{\partial y^{*}}+\left(\frac{D}{2 L_{T B}}\right) \frac{\partial w^{*}}{\partial z^{*}}=0$

where $F r_{v_{d}}=\frac{v_{d}}{\sqrt{g D}}, R e_{v_{d}}=\frac{\rho_{L} v_{d} D}{\mu_{L}}$

On the gas-liquid interface, the following conditions are applied:

$$
\begin{gathered}
0=\frac{1}{F r_{v_{d}}^{2}} g_{x}{ }^{*}-\frac{\partial p^{*}}{\partial x^{*}}+\frac{1}{R e_{v_{d}}}\left[4 \frac{\partial^{2} u^{*}}{\partial x^{* 2}}+4 \frac{\partial^{2} u^{*}}{\partial y^{* 2}}+\left(\frac{D}{L_{T B}}\right)^{2} \frac{\partial^{2} u^{*}}{\partial z^{* 2}}\right]+\frac{1}{F r_{v_{d}}^{2} E o} \sigma_{x}{ }^{*} K^{*} \\
0=\frac{1}{F r_{v_{d}}^{2}} g_{y}{ }^{*}-\frac{\partial p^{*}}{\partial y^{*}}+\frac{1}{R e_{v_{d}}}\left[4 \frac{\partial^{2} v^{*}}{\partial x^{* 2}}+4 \frac{\partial^{2} v^{*}}{\partial y^{* 2}}+\left(\frac{D}{L_{T B}}\right)^{2} \frac{\partial^{2} v^{*}}{\partial z^{* 2}}\right]+\frac{1}{F r_{v_{d}}^{2} E o} \sigma_{y}{ }^{*} K^{*} \\
\left(\frac{D}{L_{T B}}\right)\left(\frac{\partial w^{*}}{\partial t^{*}}+w^{*} \frac{\partial w^{*}}{\partial z^{*}}\right) \\
=\frac{1}{F r_{v_{d}}^{2}} g_{z}{ }^{*}-\left(\frac{D}{L_{T B}}\right) \frac{\partial p^{*}}{\partial z^{*}}+\frac{1}{R e_{v_{d}}}\left[4 \frac{\partial^{2} w^{*}}{\partial x^{* 2}}+4 \frac{\partial^{2} w^{*}}{\partial y^{* 2}}+\left(\frac{D}{L_{T B}}\right)^{2} \frac{\partial^{2} w^{*}}{\partial z^{* 2}}\right] \\
+\frac{1}{F r_{v_{d}}^{2} E o} \sigma_{z}^{*} K^{*}
\end{gathered}
$$

where $E o=\frac{g \rho_{L} D^{2}}{\sigma}$. 
In order to perform order of magnitude analysis to the dimensionless governing equations, the following orders are introduced:

$$
\begin{aligned}
& x^{*}=o(1), y^{*}=o(1), z^{*}=o(1), w^{*}=o(1), t^{*}=o(1), g_{x}^{*}=o\left(\frac{D}{L_{T B}}\right) \\
& g_{y}{ }^{*}=o\left(\frac{D}{L_{T B}}\right), g_{z}{ }^{*}=o(1), K^{*}=o(1), \sigma_{x}^{*}=o(1), \sigma_{y}^{*}=o(1), \sigma_{z}^{*}=o(1)
\end{aligned}
$$

Applying the orders given in (1-13) to the dimensionless form of continuity equation would show that $u^{*}$ and $v^{*}$ should be of order $o\left(\frac{D}{L_{T B}}\right)$ in order to keep the continuity equation intact. Similarily, introducing the same orders to the dimensionless form of momentum equations in all directions would deduce that in order to keep the inertia terms and the viscous terms of the same order of magnitude, $F r_{v_{d}}$ should be of order $o(1)$ and $R e_{v_{d}}$ should be of order $o\left(\frac{L_{T B}}{D}\right)$. Applying the same analysis to the boundries (equations (1-10) to (1-12)) would show that Eötvös number $(E o)$ should be of order $o\left(\frac{L_{T B}}{D}\right)$ and $F r_{v_{d}}$ should be of order $o(1)$ as to ensure that the surface tension force is of the same order of magnitude as the inertia force.

Regarding the near-horizontal case, $\left(70^{\circ}<\theta<90^{\circ}\right)$, a characteristic velocity $\left(v_{s}\right)$ is chosen according to a way that would treat the flow in such cases, where low values of Reynolds number $\left(R e_{v_{s}}=\frac{v_{s} D}{v}\right)$ exists.

Introducing the following dimensionless variables:

$$
\begin{aligned}
& \hat{u}=\frac{u}{v_{s}}, \hat{v}=\frac{v}{v_{s}}, \widehat{w}=\frac{w}{v_{S}}, \hat{p}=\frac{p}{\rho_{L^{v_{S}}}{ }^{2}}, x^{*}=\frac{x}{(D / 2)}, y^{*}=\frac{y}{(D / 2)}, z^{*}=\frac{z}{L_{T B}}, \\
& \hat{t}=t\left(\frac{v_{S}}{L_{T B}}\right), g_{x}{ }^{*}=\frac{g_{x}}{g}, g_{y}{ }^{*}=\frac{g_{y}}{g}, g_{z}{ }^{*}=\frac{g_{z}}{g}, \sigma_{x}{ }^{*}=\frac{\sigma_{x}}{\sigma}, \sigma_{y}^{*}=\frac{\sigma_{y}}{\sigma}, \sigma_{z}^{*}=\frac{\sigma_{z}}{\sigma}, \\
& K^{*}=\frac{K}{\left(1 / D^{2}\right)} \text { with } v_{s}=\frac{\mu_{L}}{L_{T B} \rho_{L}}
\end{aligned}
$$

The dimensionless form of contiuity and Navier stokes equations could be summarized as follow:

$$
\frac{\partial \hat{u}}{\partial x^{*}}+\frac{\partial \hat{v}}{\partial y^{*}}+\left(\frac{D}{2 L_{T B}}\right) \frac{\partial \widehat{w}}{\partial z^{*}}=0
$$




$$
\begin{gathered}
\left(\frac{D}{2 L_{T B}}\right)^{2} \frac{\partial \hat{u}}{\partial \hat{t}}+\left(\frac{D}{2 L_{T B}}\right) \hat{u} \frac{\partial \hat{u}}{\partial x^{*}}+\left(\frac{D}{2 L_{T B}}\right) \hat{v} \frac{\partial \hat{u}}{\partial y^{*}}+\left(\frac{D}{2 L_{T B}}\right)^{2} \widehat{w} \frac{\partial \hat{u}}{\partial z^{*}} \\
=\left[\left(\frac{g L_{T B}{ }^{3}}{v_{L}{ }^{2}}\right)\left(\frac{D}{2 L_{T B}}\right)^{2}\right] g_{x}^{*}-\left(\frac{D}{2 L_{T B}}\right) \frac{\partial \hat{p}}{\partial x^{*}}+\left[\frac{\partial^{2} \hat{u}}{\partial x^{* 2}}+\frac{\partial^{2} \hat{u}}{\partial y^{* 2}}+\left(\frac{D}{L_{T B}}\right)^{2} \frac{\partial^{2} \hat{u}}{\partial z^{* 2}}\right] \\
\left(\frac{D}{2 L_{T B}}\right)^{2} \frac{\partial \hat{v}}{\partial \hat{t}}+\left(\frac{D}{2 L_{T B}}\right) \hat{u} \frac{\partial \hat{v}}{\partial x^{*}}+\left(\frac{D}{2 L_{T B}}\right) \hat{v} \frac{\partial \hat{v}}{\partial y^{*}}+\left(\frac{D}{2 L_{T B}}\right)^{2} \widehat{w} \frac{\partial \hat{v}}{\partial z^{*}} \\
=\left[\left(\frac{g L_{T B}{ }^{3}}{v_{L}{ }^{2}}\right)\left(\frac{D}{2 L_{T B}}\right)^{2}\right] g_{y}{ }^{*}-\left(\frac{D}{2 L_{T B}}\right) \frac{\partial \hat{p}}{\partial y^{*}}+\left[\frac{\partial^{2} \hat{v}}{\partial x^{* 2}}+\frac{\partial^{2} \hat{v}}{\partial y^{* 2}}+\left(\frac{D}{L_{T B}}\right)^{2} \frac{\partial^{2} \hat{v}}{\partial z^{* 2}}\right] \\
\left(\frac{D}{2 L_{T B}}\right)^{2} \frac{\partial \widehat{w}}{\partial \hat{t}}+\left(\frac{D}{2 L_{T B}}\right) \hat{u} \frac{\partial \widehat{w}}{\partial x^{*}}+\left(\frac{D}{2 L_{T B}}\right) \hat{v} \frac{\partial \widehat{w}}{\partial y^{*}}+\left(\frac{D}{2 L_{T B}}\right)^{2} \widehat{w} \frac{\partial \widehat{w}}{\partial z^{*}} \\
=\left[\left(\frac{g L_{T B}{ }^{3}}{v_{L}{ }^{2}}\right)\left(\frac{D}{2 L_{T B}}\right)^{2}\right] g_{z}^{*}-\left(\frac{D}{2 L_{T B}}\right) \frac{\partial \hat{p}}{\partial z^{*}}+\left[\frac{\partial^{2} \widehat{w}}{\partial x^{* 2}}+\frac{\partial^{2} \widehat{w}}{\partial y^{* 2}}+\left(\frac{D}{L_{T B}}\right)^{2} \frac{\partial^{2} \widehat{w}}{\partial z^{* 2}}\right]
\end{gathered}
$$

It can be inferred that the influential parameters are the so-called reduced Galilei number $\left(G a_{R}=\left[\left(\frac{g L_{T B}{ }^{3}}{v_{\mathrm{L}}{ }^{2}}\right)\left(\frac{D}{2 L_{T B}}\right)^{2}\right]\right)$ and the pipe diameter to bubble length ratio.

Based on the fact that the gravity and viscous effects predominate the inertia effects, the following orders can be introduced:

$$
\begin{aligned}
& x^{*}=o(1), y^{*}=o(1), z^{*}=o(1), \widehat{w}=o(1), \hat{t}=o(1), g_{x}^{*}=o\left(\frac{D}{L_{T B}}\right) \\
& g_{y}{ }^{*}=o(1), g_{z}{ }^{*}=o\left(\frac{D}{L_{T B}}\right), K^{*}=o(1), \sigma_{x}^{*}=o(1), \sigma_{y}^{*}=o(1), \sigma_{z}^{*}=o(1)
\end{aligned}
$$

Introducing the orders given in (1-19) to the dimensionless form of continuity equation would show that $\hat{u}$ and $\hat{v}$ should be of order $o\left(\frac{D}{L_{T B}}\right)$ so as to keep the continuity equation intact. Similarily, introducing the same orders to the dimensionless form of momentum equations in all directions (equations (1-16) to (1-18)) will show that the inertia terms are of order $o\left(\frac{D}{L_{T B}}\right)^{2}$, while, the viscous terms are of order $o\left(\frac{D}{L_{T B}}\right)$ in equations (1-16) and (1-17) and of order $o(1)$ in equation (1-18). Accordingly, the momentum equations in all directions can be expressed in the following form:

$$
\left(\frac{D}{2 L_{T B}}\right)^{2} \frac{\partial \hat{u}}{\partial \hat{t}}=-\left(\frac{D}{2 L_{T B}}\right) \frac{\partial \hat{p}}{\partial x^{*}}+\left[\frac{\partial^{2} \hat{u}}{\partial x^{* 2}}+\frac{\partial^{2} \hat{u}}{\partial y^{* 2}}\right]
$$


$\left(\frac{D}{2 L_{T B}}\right)^{2} \frac{\partial \hat{v}}{\partial \hat{t}}=G a_{R} g_{y}{ }^{*}-\left(\frac{D}{2 L_{T B}}\right) \frac{\partial \hat{p}}{\partial y^{*}}+\left[\frac{\partial^{2} \hat{v}}{\partial x^{* 2}}+\frac{\partial^{2} \hat{v}}{\partial y^{* 2}}\right]$

$\left(\frac{D}{2 L_{T B}}\right)^{2} \frac{\partial \widehat{w}}{\partial \hat{t}}=-\left(\frac{D}{2 L_{T B}}\right) \frac{\partial \hat{p}}{\partial z^{*}}+\left[\frac{\partial^{2} \widehat{w}}{\partial x^{* 2}}+\frac{\partial^{2} \widehat{w}}{\partial y^{* 2}}\right]$

Although, the local inertia terms have small order of magnitude $\left[\mathrm{o}\left(\frac{D}{2 L_{T B}}\right)^{2}\right]$, they are kept only to account for the time dependent nature of the equations (unsteadiness).

Hence, it can be concluded that the flow is governed solely by the reduced Galilei number $\left(\mathrm{Ga}_{\mathrm{R}}\right)$ with an order of magnitude $o(1)$.

On the gas-liquid interface, the following conditions are applied:

$$
\begin{aligned}
& 0=G a_{R} g_{x}^{*}-\left[\left(\frac{D}{2 L_{T B}}\right) \frac{\partial \hat{p}}{\partial x^{*}}\right]_{S}+\left[\frac{\partial^{2} \hat{u}}{\partial x^{* 2}}+\frac{\partial^{2} \hat{u}}{\partial y^{* 2}}\right]_{S}+\left[\frac{G a_{R}}{E o} \sigma_{x}^{*} K^{*}\right]_{S} \\
& 0=G a_{R} g_{y}^{*}-\left[\left(\frac{D}{2 L_{T B}}\right) \frac{\partial \hat{p}}{\partial y^{*}}\right]_{S}+\left[\frac{\partial^{2} \hat{v}}{\partial x^{* 2}}+\frac{\partial^{2} \hat{v}}{\partial y^{* 2}}\right]_{S}+\left[\frac{G a_{R}}{E o} \sigma_{y}^{*} K^{*}\right]_{S} \\
& \left(\frac{D}{2 L_{T B}}\right)^{2} \frac{\partial \widehat{w}}{\partial \hat{t}}+\left(\frac{D}{2 L_{T B}}\right) \hat{u} \frac{\partial \widehat{w}}{\partial x^{*}}+\left(\frac{D}{2 L_{T B}}\right) \hat{v} \frac{\partial \widehat{w}}{\partial y^{*}}+\left(\frac{D}{2 L_{T B}}\right)^{2} \widehat{w} \frac{\partial \widehat{w}}{\partial z^{*}} \\
& =G a_{R}\left[g_{z}{ }^{*}+\frac{1}{E o} \sigma_{z}{ }^{*} k^{*}-\left[\left(\frac{D}{2 L_{T B}}\right) \frac{\partial \hat{p}}{\partial z^{*}}\right]_{S}+\left[\frac{\partial^{2} \widehat{w}}{\partial x^{* 2}}+\frac{\partial^{2} \widehat{w}}{\partial y^{* 2}}\right]_{S}\right.
\end{aligned}
$$

The same orders can be used for the boundaries to show that if the surface tension is assumed to have the same order as the gravity term, this implies that Eötvös number (Eo) should be of order $o\left(\frac{L_{T B}}{D}\right)$.

When the pipe is approximately horizontal, that is for $\theta \approx 90^{\circ}$, with the following conditions: the absence of the axial pressure gradient $\left(\frac{\partial \hat{p}}{\partial z^{*}}\right)$, and the bubble is approxiametly stagnant axially $(\widehat{w} \approx 0)$, the governing equations reduce to the following form:

$$
\begin{aligned}
& \left(\frac{D}{2 L_{T B}}\right)^{2} \frac{\partial \hat{u}}{\partial \hat{t}}=-\left(\frac{D}{2 L_{T B}}\right) \frac{\partial \hat{p}}{\partial x^{*}}+\left(\frac{\partial^{2} \hat{u}}{\partial x^{* 2}}+\frac{\partial^{2} \hat{u}}{\partial y^{* 2}}\right) \\
& \left(\frac{D}{2 L_{T B}}\right)^{2} \frac{\partial \hat{v}}{\partial \hat{t}}=G a_{R} g_{y}^{*}-\left(\frac{D}{2 L_{T B}}\right) \frac{\partial \hat{p}}{\partial y^{*}}+\left(\frac{\partial^{2} \hat{v}}{\partial x^{* 2}}+\frac{\partial^{2} \hat{v}}{\partial y^{* 2}}\right)
\end{aligned}
$$


$\frac{\partial \hat{u}}{\partial x^{*}}+\frac{\partial \hat{v}}{\partial y^{*}}=0$

The limiting case when the bubble is stagnant in a entirely horizontal pipe $\left(\theta=90^{\circ}\right)$ gives the following equations for the boundaries:

$0=\rho_{L} g_{x}-\frac{\partial p}{\partial x}+K \sigma_{x}$
$0=\rho_{L} g_{y}-\frac{\partial p}{\partial y}+K \sigma_{y}$

The following dimensionless variables can be used to perform dimensionless analysis for the governing equations in the horizontal pipe case:

$$
\begin{aligned}
& x^{*}=\frac{x}{(D / 2)}, y^{*}=\frac{y}{(D / 2)}, \bar{p}=\frac{p}{\rho_{L} g \frac{D}{2}}, g_{x}{ }^{*}=\frac{g_{x}}{g}, g_{y}{ }^{*}=\frac{g_{y}}{g}, \sigma_{x}{ }^{*}=\frac{\sigma_{x}}{\sigma}, \\
& \sigma_{y}^{*}=\frac{\sigma_{y}}{\sigma}, K^{*}=\frac{K}{\left(1 / D^{2}\right)}
\end{aligned}
$$

The final form of the dimensionless governing equations for the horizontal pipe case is given by:

$$
\begin{aligned}
& 0=g_{x}{ }^{*}-\frac{\partial \bar{p}}{\partial x^{*}}+\frac{K^{*} \sigma_{x}{ }^{*}}{E o} \\
& 0=g_{y}{ }^{*}-\frac{\partial \bar{p}}{\partial y^{*}}+\frac{K^{*} \sigma_{y}{ }^{*}}{E o}
\end{aligned}
$$

In conclusion, it is obvious that equations (1-32) and (1-33) govern the static pressure distribution around the stagnant deformed bubble. The main governing parameter that regulates the pressure filed is Eötvös number, where Galilei number also ceases to be effective, and Reynolds and Froude numbers are no longer influencing parameters. 
Table 1- Simulation cases used to explore the effect of both reduced Galilei number $\left(G a_{R}\right)$ and Eötvös number $(E o)$ on dynamics of Taylor bubble for inclination angle range of $70^{\circ}<\theta<90^{\circ}$, with their corresponding results.

\begin{tabular}{|c|c|c|c|c|c|}
\hline $\begin{array}{l}\text { Case } \\
\text { number }\end{array}$ & $\begin{array}{l}\text { Inclination } \\
\text { angle (degrees) }\end{array}$ & $\begin{array}{l}\text { Reduced Galilei } \\
\text { number }\left(\boldsymbol{G} a_{R}\right)\end{array}$ & $\begin{array}{c}\text { Eötvös } \\
\text { number }(E o)\end{array}$ & $\begin{array}{c}\text { Froude } \\
\text { number } \\
\left(\boldsymbol{F} \boldsymbol{r}_{v_{d}}\right)\end{array}$ & $\left(\frac{L_{T B}}{D}\right)_{p}$ \\
\hline \multirow{2}{*}{1} & 75 & 15 & \multirow{4}{*}{66} & 0.0491 & 3.893 \\
\hline & 85 & 15 & & 0.0262 & 5.053 \\
\hline \multirow{2}{*}{2} & 75 & \multirow{2}{*}{200} & & 0.1602 & 3.680 \\
\hline & 85 & & & 0.0916 & 5.032 \\
\hline \multirow{2}{*}{3} & 75 & \multirow{4}{*}{200} & \multirow{2}{*}{66} & 0.1602 & 3.680 \\
\hline & 85 & & & 0.0916 & 5.032 \\
\hline \multirow{2}{*}{4} & 75 & & \multirow{2}{*}{100} & 0.1696 & 3.922 \\
\hline & 85 & & & 0.1041 & 5.505 \\
\hline
\end{tabular}

Table 2- Summary of the main correlations proposed in the literature for the rising velocity of a Taylor bubble in inclined pipes with stagnant liquid.

\begin{tabular}{|c|c|c|}
\hline $\begin{array}{l}\text { Reference/ } \\
\text { Type of } \\
\text { study } \\
\end{array}$ & Correlation & Assumptions and comments \\
\hline $\begin{array}{l}\text { Bendiksen } \\
(1985) \\
\text { Experimental } \\
\text { and } \\
\text { theoretical } \\
\end{array}$ & $v_{d}=v_{d}^{h} \cos \theta+v_{d}^{v} \sin \theta, v_{d}^{h}=0.351 \sqrt{g D}, v_{d}^{v}=0.542 \sqrt{g D}$ & $\begin{array}{l}\text { - } \theta \text { ranges from }-90^{\circ} \text { to } 90^{\circ} . \\
\text { - } E \text { o ranges from } 50-340, M \approx 2.63 \cdot 10^{\circ} \\
\text { - } F r_{v_{\text {max }}} \text { at } 40^{\circ}<\theta<60^{\circ}\end{array}$ \\
\hline $\begin{array}{l}\text { Weber et al. } \\
(1986) \\
\text { Experimental }\end{array}$ & $\begin{array}{l}F r_{v_{d}}=F r_{v_{d}}{ }^{h} \cos \theta+F r_{v_{d}}{ }^{v} \sin \theta+Q, \\
Q=\left\{\begin{array}{cc}1.37\left(\Delta F r_{v_{d}}\right)^{\frac{2}{3}} \sin \theta(1-\sin \theta), & \text { if } \Delta F r_{v_{d}}>0 \\
0, & \text { if } \Delta F r_{v_{d}} \leq 0\end{array}\right.\end{array}$ & $\begin{array}{l}\text { - } \theta \text { ranges from } 0 \text { to } 90^{\circ} \text { from } \\
\text { horizontal position. } \\
\text { - } E \text { o ranges from } 4.9-490, M=2.2 \cdot 10^{-11} \\
-1.5 \cdot 10^{4} \\
\text { - } F r_{v_{\text {max }}} \text { at } 30^{\circ}<\theta<60^{\circ} \\
\text { - High-viscosity Newtonian liquids. }\end{array}$ \\
\hline $\begin{array}{l}\text { Hasan and } \\
\text { Kabir (1988) } \\
\text { Experimental } \\
\text { and } \\
\text { theoretical }\end{array}$ & $v_{d}=v_{d}^{v} \sqrt{\sin \theta}(1+\cos \theta)^{1.2}, v_{d}^{v}=0.35 \sqrt{g D}, v_{d}^{h}=0$ & $\begin{array}{l}\text { - } \theta \text { ranges from } 30 \text { to } 90^{\circ} \text { from } \\
\text { horizontal position. } \\
\text { - } A s s u m e v_{d}^{h}=0 \text {. } \\
\text { - Applicable for systems with large } N_{f} \\
\text { and } E o \text { values } \\
\text { - } E o=2200, M=2.63 \cdot 10^{-11}\end{array}$ \\
\hline $\begin{array}{l}\text { Petalas and } \\
\text { Aziz (2000) } \\
\text { Theoretical }\end{array}$ & 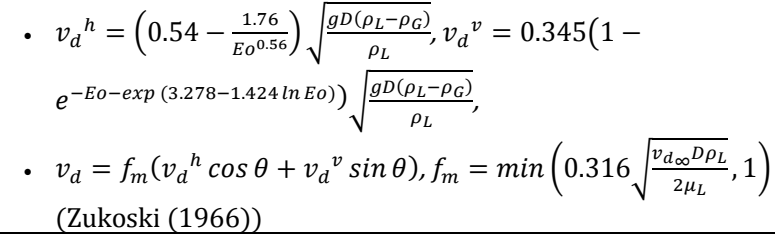 & $\begin{array}{l}\text { - } \theta \text { ranges from }-90^{\circ} \text { to } 90^{\circ} \text {. } \\
\text { - } F r_{v_{d_{\text {max }}}} \text { at } 40^{\circ} \\
\text { - Applicable for high Reynolds numbers }\end{array}$ \\
\hline $\begin{array}{l}\text { Gokcal et al. } \\
\text { (2009) } \\
\text { Experimental }\end{array}$ & $\begin{array}{l}\text { - } h / D=0.1038 \ln \mu_{L}+0.9684, S / D=\sqrt{1-(2 h / D-1)^{2}} \\
\text { - } \gamma_{1}=\left\{\begin{array}{l}\pi-\sin ^{-1}(S / D) \text { if } h / D<0.5 \\
\sin ^{-1}(S / D) \text { if } h / D \geq 0.5^{\prime}\end{array}\right. \\
\text { - } \gamma=\min \left(\gamma_{1}, 1.444784\right), \zeta=(\gamma-0.5 \sin 2 \gamma) / \pi \\
\text { - } \Delta=\frac{1+\zeta}{\zeta}\left(\left(\frac{D}{2}(1-\cos \gamma)\right)-\left(\frac{\left(\frac{D}{2}(1-(1-\zeta) \cos \gamma)\right)+\frac{2}{3 \pi} \sin \gamma}{1-\zeta^{2}}\right)\right) \times 2.2 \\
\text { - } v_{2}=\sqrt{g D\left((1-(1-\zeta) \cos \gamma)-\frac{2}{3 \pi} \sin ^{3} \gamma\right)-2 \Delta g(1-\zeta)}\end{array}$ & $\begin{array}{l}\text { - } \theta \text { ranges from } 5 \text { to } 90^{\circ} \text { measured } \\
\text { from the horizontal position. } \\
\text { - } E o=776, M=4.52 \cdot 10^{-10}-1.63 \cdot 10^{3} \\
\text { - } F r_{v_{\text {max }} \text { at } 40^{\circ} \text {. }} \\
\text { - Correlation applicable for high viscosity } \\
\text { oil. }\end{array}$ \\
\hline
\end{tabular}




\begin{tabular}{|c|c|c|}
\hline & $\begin{array}{l}\text { - } v_{d}^{h}=v_{1}=(1+\zeta) v_{2}, v_{d}^{v}=\frac{-8}{3} \frac{\mu_{L}}{\rho_{L} D}+\sqrt{\frac{2}{9} g D+\frac{64}{9}\left(\frac{\mu_{L}}{\rho_{L} D}\right)^{2}} \\
\text { - } v_{d}=v_{d}^{h}(\cos \theta)^{1.5}+v_{d}^{v}(\sin \theta)^{0.7}\end{array}$ & \\
\hline $\begin{array}{l}\text { Jeyachandra et } \\
\text { al. (2012) } \\
\text { Experimental }\end{array}$ & $\begin{array}{l}F r_{v_{d}}=F r_{v_{d}}{ }^{h} \cos \theta+F r_{v_{d}}{ }^{v} \sin \theta \\
F r_{v_{d}}{ }^{h}=0.53 \exp \left(-13.7 N_{f}^{-0.46} E o^{-0.1}\right) \\
F r_{v_{d}}{ }^{v}=\frac{-8}{3} N_{f}^{-1}+\sqrt{\frac{2}{9} \frac{\rho_{L}}{\left(\rho_{L}-\rho_{G}\right)}+\frac{64}{9} N_{f}^{-2}}\end{array}$ & $\begin{array}{l}\text { - } \theta \text { ranges from } 0 \text { to } 90^{\circ} \text { measured } \\
\text { from the horizontal position } \\
\text { - } E o=776-6985, M=0.254-49.1 \\
\text { - } F r_{v_{\text {max }}} \text { at } 30^{\circ}<\theta<50^{\circ}\end{array}$ \\
\hline $\begin{array}{l}\text { Moreiras et al. } \\
\text { (2014) } \\
\text { Experimental }\end{array}$ & 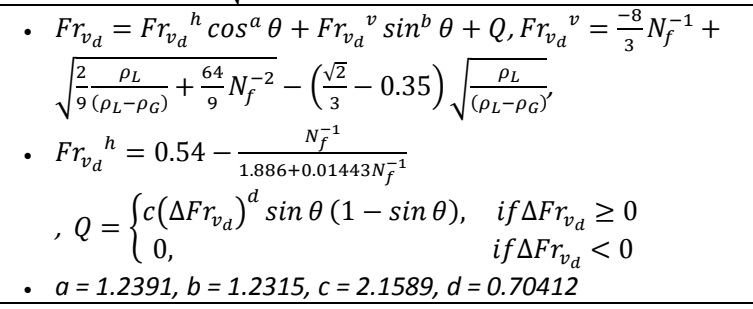 & $\begin{array}{l}\text { - } \theta \text { ranges from } 0 \text { to } 90^{\circ} \text { measured } \\
\text { from the horizontal position } \\
\text { - } E o=804, M=1.25 \cdot 10^{-3}-10.412 \\
\text { - } F r_{v_{\text {dmax }}} \text { at } 30^{\circ}<\theta<50^{\circ}\end{array}$ \\
\hline $\begin{array}{l}\text { Lizarraga- } \\
\text { García (2016) } \\
\text { Experimental } \\
\text { and } \\
\text { Numerical } \\
\text { work }\end{array}$ & $\begin{aligned} & \text { - } F r_{v_{d}}{ }^{v}=\frac{0.34 /\left(1+(14.793 / E o)^{3.06}\right)^{0.58}}{\left(1+\left(\left(\frac{N_{f}}{31.08\left(1+(29.868 / E o)^{1.96}\right)^{0.49}}\right)\right)^{a}\right)^{b}}, a=-1.45(1+ \\
&\left.(24.867 / E o)^{9.93}\right)^{0.094}, b=-1.0295 / a \\
& \text { - } F r_{v_{d}}= \\
& F r_{v_{d}}{ }^{v}(1-\exp (-B((E o, M)) \theta))(1+(C(E o, M)) \sin (2 \theta)) \\
& \text { - } B(E o, M)=47.06 F r_{v_{d}}{ }^{v}+4 \\
& \text { - } C(E o, M)= \\
&-0.9118 F r_{v_{d}}{ }^{v}+0.67+ \\
& \frac{\left(-0.0148\left(\log _{10} M\right)^{2}+0.125 \log _{10} M+0.9118 F r_{v_{d}}{ }^{v}+1.118\right)}{\left(1+(E o / 20)^{8}\right)^{8}} \\
&\end{aligned}$ & $\begin{array}{l}\text { - } \theta \text { ranges from } 5 \text { to } 90^{\circ} \text { measured } \\
\text { from the horizontal position. } \\
\text { - The horizontal pipe case is not } \\
\text { included. } \\
\text { - } E o=10: 700, M=1 \cdot 10^{-6}-5 \cdot 10^{3} \\
\text { - } F r_{v_{\text {max }}} \text { at } 45^{\circ} \text {. }\end{array}$ \\
\hline
\end{tabular}

Table 3- Test matrix for simulations of drift of Taylor bubble through stagnant liquid in inclined pipes based on the experimental work of Shosho and Ryan (2001).

\begin{tabular}{ccccccc} 
Case Number & Working fluid & $\mathrm{D}(\mathbf{m})$ & $\begin{array}{c}\text { Density } \\
\left(\boldsymbol{K} \boldsymbol{g} / \boldsymbol{m}^{3}\right)\end{array}$ & $\begin{array}{c}\text { Viscosity } \\
(\boldsymbol{P a} \boldsymbol{s})\end{array}$ & $\begin{array}{c}\text { Surface tension } \\
(\boldsymbol{N} / \boldsymbol{m})\end{array}$ & Eo \\
\hline \hline 1 & 60\% Corn syrup & $1.27 \times 10^{-2}$ & 1195.75 & $3.60 \mathrm{E}-02$ & 0.049 & 38.61 \\
\hline 2 & $80 \%$ Corn syrup & $2.54 \times 10^{-2}$ & 1320.02 & $1.91 \mathrm{E}-01$ & 0.051 & 163.8 \\
\hline \hline
\end{tabular}

